\title{
Remediation of PTSD in a Combat Veteran: A Case Study
}

\author{
George Lindenfeld ${ }^{*}$, George Rozelle², John Hummer ${ }^{3}$, Michael R. Sutherland4, and James C. \\ Miller $^{5}$
}

${ }^{1}$ Reset Therapy Professional Institute, LLC, Sarasota, Florida \& Hendersonville, North Carolina, USA

${ }^{2}$ MindSpa Integrative Wellness Center, Sarasota, Florida, USA

3James H. Quillen VA Medical Center, Johnson City, Tennessee, USA

${ }^{4}$ University of Massachusetts, Amherst, Massachusetts, USA

${ }^{5}$ U.S. Air Force Research Laboratory, Corpus Christi, Texas, USA

\begin{abstract}
The posttraumatic stress disorder (PTSD) condition is a systemic neuroinflammatory state that emanates from a failure to recover from traumatic occurrence(s). Major complications associated with PTSD include problems with impulse control and issues related to verbal and physical outbursts of anger and rage. The Veteran's Administration (VA) projects a post-9/11 veteran population of around 3.5 million by 2019 . Emotional problems are prevalent among combat service members and veterans with about half of the group suffering from various symptoms of PTSD. Three in four among them report they are reliving traumas in the form of flashbacks and nightmares. Current mental health treatments have not fully remediated the negative impact that results from PTSD. We present a case study of a novel and transformative treatment approach called Reconsolidation Enhancement by Stimulation of Emotional Triggers (RESET) Therapy. The intervention uses binaural sound to unlock the memory reconsolidation process, thereby releasing the emotional component of experienced trauma. RESET Therapy offers a compelling therapeutic adjunct to the practicing biofeedback/neurofeedback clinician, who is under constant pressure to deliver interventions that are rapid, tolerable, and cost-effective. Additionally, the treatment spares the therapist from repeated exposures to the raw limbic activity of traumatized patients, thereby minimizing the potential for vicarious traumatization.
\end{abstract}

Keywords: posttraumatic stress disorder; trauma; memory reconsolidation; binaural sound; the neuronal network of PTSD

Citation: Lindenfeld, G., Rozelle, G., Hummer, J., Sutherland, M. R., \& Miller, J. C. (2019). Remediation of PTSD in a combat veteran: A case study. NeuroRegulation, 6(2), 102-125. https://doi.org/10.15540/nr.6.2.102

*Address correspondence to: Dr. George Lindenfeld, Glenhouse Drive GL-327, Sarasota, FL 34231, USA. Email: glindy123@gmail.com

Edited by:

Rex L. Cannon, PhD, SPESA Research Institute, Bloomfield Hills, Michigan, USA; Knoxville Neurofeedback Group, Knoxville, Tennessee, USA

Copyright: @ 2019. Lindenfeld et al. This is an Open Access article distributed under the terms of the Creative Commons Attribution License (CC-BY).

\section{Reviewed by:}

Randall Lyle, PhD, Mount Mercy University, Cedar Rapids, lowa, USA

Nancy L. Wigton, PhD, Grand Canyon University, Phoenix, Arizona, USA

The included case study aims to introduce the neurofeedback practitioner to a promising new neurofeedback-based intervention called Reconsolidation Enhancement by Stimulation of Emotional Triggers (RESET) Therapy, a type of brief nonverbal exposure therapy utilizing auditory stimulation via an individually-intonated binaural beat. We demonstrate the RESET procedure using a case study involving a veteran volunteer who took part in a larger pilot study (Lindenfeld, Rozelle, Soutar, Hummer, \& Sutherland, 2019).

\section{Background}

Psychological trauma inflicts a shock to the central nervous system that results in a reduction in connectivity between limbic and cortical processes. The varied consequences reverberate throughout the brain over time depending upon the patient's previous history, physiological status, and the ongoing social distress encountered. Severe anxiety and depression are likely to be concomitant states which vary over time and circumstance, reflected in hemispheric asymmetries. The 
posttraumatic stress disorder (PTSD) condition is a systemic neuroinflammatory disorder that emanates from a failure among many human beings to recover from traumatic occurrence(s). Inability to recover from trauma can occur following the experience of stress-induced "fight, flight, or freeze" event(s). Unlike other mammalian species who can "shake it off," humans may lock recall of their adverse experience into the long-term memory system of the brain and body (Levine \& Frederick, 1997).

Psychological symptoms of PTSD are accompanied by a neuroinflammatory process in the brain and body, where oxidative stress and excitotoxicity takes an adverse toll over time (Soutar, Hopson, \& Longo, 2016; Bam et al., 2016). Soutar et al. (2016) outlined successive stages of the oxidative stress process and its adverse effects upon brain activation patterns as depicted via a series of quantitative electroencephalograms (qEEGs). Patients with PTSD vary in their stage of the stress response, as reflected in their brain physiology (Soares, Marques, Magalhães, Santos, \& Sousa, 2014). Any effort to oversimply PTSD by looking for a singular qEEG brainwave "signature" misses the condition's variability point, as it reflects a failure to grasp the dynamic and degrading nature of the stress response syndrome over time.

Presently, there is no single unified or encompassing model of PTSD. It is generally accepted, however, that individuals with chronic PTSD encounter difficulties in the regulation of attention and arousal, self or other emotional awareness, and social-emotional processing. These processes depend upon the functional integrity of large-scale brain networks encompassing cortical, subcortical, and brainstem structures.

We begin the article by summarizing some of the current neuroscience understanding of PTSD, followed by a limited review of psychological, biofeedback, and neurofeedback research addressing the treatment of PTSD. We present a heuristic neuronal model of PTSD to provide testable hypotheses for the clinician using the RESET method, and then present our case study of a veteran successfully treated with RESET to demonstrate the method and how it can be replicated via future research.

\section{Remediation of PTSD}

Remediation of PTSD is an ongoing topic of discussion among many mental health practitioners because we have been unable to restore the impacted individual to a full state of prior functioning.
While advised by leading governmental agencies such as the National Institute of Mental Health $(\mathrm{NIMH})$ in the use of frontline therapies, we are still seemingly not able to meet the demand of those most impacted by the condition. As noted in the NIMH (2019) description of treatments for PTSD, the primary interventions discussed are talk based psychotherapy, medications, or both. With the Veteran's Administration (VA) projecting numbers in the millions of those affected by PTSD, (Tanielian \& Jaycox, 2008) finding better answers to enable mental health professionals to restore those altered by PTSD are critical.

A recent letter to the editor Krystal et al. (2017) reported a 2017 consensus statement of the PTSD Psychopharmacology Working Group. They advise that "there seems to be no visible horizon for advancements in medications that treat symptoms or enhance outcomes in persons with a diagnosis of PTSD" (p. e51). A follow-up letter by another group of researchers, Lipov, Tukan, and Candido (2018), comments that "with only $50 \%$ of veterans seeking care and a $40 \%$ recovery rate, current strategies will effectively reach no more than $20 \%$ of all veterans who need PTSD treatment" (p. e17).

\section{Neuroscience Underpinnings}

Rauch, Shin, and Phelps (2006) outlined a neurocircuitry model of PTSD that emphasizes the role of the amygdala, as well as its interactions with the ventral/medial prefrontal cortex including but not limited to the anterior cingulate, prefrontal cortex, orbitofrontal cortex, and hippocampus. Rauch et al. (2006) proposes a diathesis-stress model involving a fear-conditioning process that could include any possible combination of amygdala hyperresponsivity, ventromedial prefrontal cortex deficiency, hippocampal deficiency, or exaggerated sensitivity or susceptibility to the effects of stress. They cite earlier fMRI studies that showed the right amygdala hyperactivity and decreased left prefrontal, Broca's area activity. Brain imaging research has tended to provide evidence that an overactive right hemisphere is involved in persistent posttraumatic stress (Engdahl et al., 2010; Engels et al., 2007; Lanius, Frewen, Tursich, Jetly, \& McKinnon, 2015; Rauch et al., 2006). Underarousal of the left prefrontal/frontal cortex has been demonstrated in depression (Davidson \& Hugdahl, 1996; Herrington et al., 2010). Broca's area becomes underactive in psychological trauma, speechless horror, followed by fragmentation of, or lack of, verbal story or narrative as noted by van der Kolk, McFarlane, and Weisaeth (1996). 
At least three large-scale brain networks-also referred to as intrinsic connectivity networks-have been implicated in psychiatric disorders, including PTSD. These include the default mode network (DMN), salience network, and central executive control network (Lanius et al., 2015). Networks, both large-scale and mesoscale, tend to be bihemispheric (Laird et al., 2011). Network activity has been established via functional neuroimaging (e.g., fMRI) studies.

Resting-state conditions are observed through changes in blood flow in the brain, assessed via the blood-oxygen-level-dependent (BOLD) signal in fMRI. Because the brain is always active even in the absence of task involvement, any brain region will have spontaneous fluctuations in the BOLD signal. The resting state is used to assess the brain's functional organization connectivity or communication level and to evaluate whether it is altered in neurological and psychiatric conditions (Sporns, 2010). Functional connectivity lasting seconds is reflected by varied brain region intercommunication that share functional properties and is an expression of network behavior underlying high-level cognitive functions. These networks increase and decrease in activation in proportional and antagonistic manners during the performances of emotional and cognitive tasks.

Default Mode Network (DMN). The DMN involves the anterior and posterior medial cortices and the lateral parietal lobes, and it is most active when an individual is in a waking, resting state. It activates an internal focus, such as daydreaming, retrieving autobiographical information, self-monitoring, inner reflection upon one's emotional state, imagining the future, focus upon personal values and morals, and theory of mind thinking about others, wondering socially about the perceptions, feelings, and motivations of others. The default state encompasses emotional reasoning, social judgment, episodic memory recalling specific events in time, and story comprehension understanding and remembering a narrative. The $\mathrm{DMN}$ also focuses on future goals and internally valued rewards.

The DMN has been hypothesized to be relevant to disorders including Alzheimer's disease, autism, schizophrenia, depression, chronic pain, PTSD, among other disorders (Menon, 2015; Sporns, 2010). Lower connectivity between brain regions has been found across the DMN in persons with long-term trauma such as childhood abuse or neglect and disrupted attachment. In PTSD, lower activation has been found in the posterior cingulate gyrus, and in severe posttraumatic stress (PTS), lower overall connectivity within the DMN. If the DMN is altered, it changes the way one perceives events, one's social and moral reasoning, and makes a person more susceptible to major depression-like symptoms.

Salience Network. The salience (vigilance) network $(\mathrm{SN})$, which involves the dorsal anterior cingulate and frontoinsular cortex, is involved in maintaining a sustained state of alertness, involving the right frontal and right parietal lobes. As opposed to the narrow, task-focused attention of the left hemisphere, the right hemisphere employs a broader and more vigilant orientation to the environmental, allowing rapid detection of the location of a stimulus-especially an infrequent or unexpected stimulus (Posner \& Raichle, 1994; McGilchrist, 2009), it plays a crucial role in salience detection-directing behavior to the most pertinent actions. The anterior insula of the $\mathrm{SN}$ is thought to meditate the engagement of the central executive network (CEN) and disengagement of the DMN, and hence the dynamic interplay between the externally and internally focused attention and cognitiveaffective processing.

Central Executive Network (CEN). The CEN involves the dorsolateral prefrontal cortex and the posterior parietal cortex. It is responsible for highlevel cognitive functions, notably the control of attention and working memory. It acts as a controller that directs the engagement of other areas that contribute sensory or conceptual content to network operations. For example, coordination of the prefrontal and posterior parietal areas channels the flow of sensory and motor activity to prepare for perceptual-motor processing which involves the dorsolateral prefrontal cortex and the posterior parietal cortex; it is critical to verbal learning and executive functioning (Bressler \& Menon, 2010).

Lanius et al. (2015) reviewed experimental neuroimaging research demonstrating disrupted internetwork activity in PTSD, as opposed to healthy controls. When given a working memory task, healthy controls readily shifted to engage the CEN, whereas the PTSD group showed difficulty shifting out of DMN activity. By contrast, when the task involved autobiographical memory retrieval, the PTSD group showed decreased recruitment of DMN networks, relative to healthy controls. Memories recalled in first-person perspective elicited greater medial temporal amygdala activity than third-person perspective. Interestingly, the emotional intensity of the memory was associated with increased frontal- 
parietal CEN activity in healthy controls but decreased frontal-parietal activity within the CEN among PTSD participants. There was a positive correlation between dissociative experiences and communication between the DMN and CEN in PTSD patients.

While healthy controls engage the CEN on workingmemory tasks, PTSD subjects engage brain regions irrelevant to the task, such as the DMN, engaging in self-referential processes rather than outwardly directed task behavior, signaling cognitive dysfunction. Findings demonstrate that the ability to engage and shift between task-relevant CEN and task-irrelevant DMN brain networks during processing tasks which are partly mediated by the anterior insula is impaired in PTSD (Lanius et al., 2015). A brain that is dysregulated from trauma cannot recall, evaluate, explain, plan, or coordinate complex cognitive and emotional processes (Soutar, in press).

\section{Synthesis of Findings}

From the research above, we can synthesize the following findings: the amygdala shows increased activation, which translates to overactivation of the fear circuit with exaggerated fight or flight responses, in PTSD. The prefrontal cortex shows decreased activation, which translates to weakening the influence of the thinking or executive brain over other areas or networks. The cingulate cortex shows decreased activation, translating to decreasing emotional regulation. The insula may show increased or decreased activation, reflecting active versus numbing states experienced in the body. The amygdala's connections to the prefrontal cortex are strong, whereas the prefrontal cortex's ability to inhibit the amygdala weakens in PTS.

In PTS, the amygdala's influence upon connections to the anterior cingulate cortex emotional regulation system is strong, whereas the anterior cingulate reciprocal connections to the amygdala are weakened. The right prefrontal and frontal cortex must increase in activation elevating amplitudes in the beta, and high beta frequency ranges to exert inhibitory control over the right amygdala. The inhibitory aspect is a form of a compensatory process (Merabet \& Pascual-Leone, 2010) characteristic of deregulated self-organizing neurobiological systems where timing has become disrupted (Othmer, Othmer, \& Kaiser, 1999) where some brain areas are running too slowly, other brain areas are running too rapidly-hence, brain too slow; brain too fast. Similarly, the right parietal cortex must increase its activation elevating amplitudes in the beta, and high beta frequency ranges to control the hyperaroused reticular activating system (RAS) in the brainstem. The delicate normal asymmetry pattern between the left and right hemispheres is now reversed, whereas the left hemisphere becomes underactive.

Swingle (2008) has noted a phenomenon called alpha blocking in PTSD. Alpha amplitudes normally increase by $30 \%$ to $60 \%$ when eyes are closed, particularly in the occipital regions. Many PTSD subjects do not show the expected normal pattern of alpha amplitude increases in the eyes-closed condition. They also tend to have lower than average theta amplitudes in the occipital areas. The phenomenon of alpha blocking has been largely based on clinical observation and practice and may require further empirical investigation.

\section{Veterans with PTSD and TBI}

About veterans with PTSD and traumatic brain injury (TBI), Brenner (2011) reviewed neuroimaging and neuropsychological data on veterans returning from Iraq and Afghanistan. Focusing specifically on PTSD research, Brenner concluded that neurobiological activation influences functioning. He noted that chronic activation of, or alteration to, structures in the limbic system and prefrontal cortex is detrimental to long-term physical and mental wellbeing. Reduced hippocampal volume and other premorbid neurobiological risk factors from exposure to developmental and premilitary stressors contribute to the development of combat-related PTSD.

\section{Hypothesis}

We hypothesize that the PTSD condition is a systemic neuroinflammatory disorder that emanates from a failure among many human beings to recover from traumatic occurrence(s). Inability to recover from trauma can happen following the experience of stress-induced fight, flight, or freeze event(s). As an example of how other mammal species who can shake it off, whereas humans may lock recall of their adverse experience into the long-term memory system of the brain and body, Levine and Frederick (1997) inform us that "tigers don't get ulcers"; thus insinuating that the predator is not vulnerable to stress as are humans.

We will proceed with clarification of different elements of our hypothesis, beginning with the systemic aspect of PTSD. By systemic, we mean the impact of an adverse effect on multiple body 
organs and tissue, systemwide. Next, we specifically discuss the neuroinflammatory component that may be initiated in response to the impact of stress. And finally, we will explain the memory component within the context of the reconsolidation process.

\section{PTSD as a Systemic Disorder}

Among Australians who served in Iraq or Afghanistan, the estimate of those incurring PTSD is $16.5 \%$. Those who served in Vietnam suffered a lifetime incidence rate of $20.9 \%$ (McLeay et al., 2017). Among these Australian veterans, many experienced accompanying physical comorbidities, including chronic disease (McLeay et al., 2017), suggesting that PTSD should not be seen as a mental condition but rather as a systemic disorder. Other investigators (Mellon, Gautam, Hammamieh, Jett, \& Wolkowitz, 2018) found increased rates of somatic comorbidities in those with PTSD in comparison to those without it, including immune dysfunction and cardiovascular disease. Their findings support the perspective that PTSD may be a systemic condition. They conclude with the position that PTSD with its accompanying comorbid conditions places affected individuals at increased mental and physical health risk.

\section{PTSD as a Neuroinflammatory Contributor}

Microglia, comprising $10 \%$ of the central nervous system (CNS) population, are the immune cells of the CNS, which play essential roles in mediating neuroinflammatory responses. Their primary function appears to be that of coordinating the interaction between the immune system and the brain. Unfortunately, their low turnover rate makes them susceptible to the proinflammatory effects of stress, injury, or age. The extent of neuroinflammation is dependent on the situation, length of time, and the intensity of the trauma effects. Chronic low-level effects trail the acute phase of trauma, leading to diminished neuronal plasticity and accompanying impairment in cognitive functioning. Increased degrees of the chronic effect are associated with actual damage to the nervous system that is target specific for neurodegenerative diseases.

Chronic or traumatic stressors promote an increased neuroinflammatory profile involving both microglia and bone marrow-derived macrophages. With the activation of the above process, the immune system is thought to relay information to the brain, which consequently promotes prolonged anxiety-like behavior. Chronic stress appears to be associated with impairment to intellectual abilities, accelerating a decline in the effectiveness of cognitive abilities (DiSabato, Quan, \& Godbout, 2016).

A 2018 systematic review of literature explored the possible association between PTSD and low-grade inflammation. Speer, Upton, Semple, and McKune (2018) found evidence for the presence of inflammatory biomarkers that were elevated across included studies in the varied PTSD groups but not in the control groups.

A recent study found that enhanced inflammatory processes across a wide range of psychiatric diagnoses are thought to disrupt neurobiological mechanisms that regulate cortical plasticity and cognition suggesting that a cross-conditional approach be considered for managing disrupted cognition in psychiatric patients (Fourrier, Singhal, \& Baune, 2019).

Martone (2019) found a correlation between an overactivated immune response and the advancement of psychiatric symptoms. He notes the presence of harmful effects on cognition and behavior, whereas subduing inflammation can considerably improve mood and sensorium. Particular brain regions that trigger alarm and arousal appear to be predominantly vulnerable to the effects of inflammation.

Sumner et al. (2017) found PTSD to be associated with heightened cardiovascular disease risk by fostering a neuroinflammatory state. They suggest that impaired endothelial function and increased inflammation may serve as a pathway through which chronic PTSD may increase a cardiovascular disease risk factor.

As substantiated above, varied investigators are increasingly exploring the long-term effects that emerge due to trauma exposure (Miller, Lin, Wolf, \& Miller, 2018). Many early-onset conditions are noted, including dementia and other neurocognitive disorders. Miller et al. (2018) report a strong association of trauma activation with related neurobiological pathways within the context of a state of heightened physiological arousal. These researchers propose that the molecular consequences of the syndrome activate elevated systemic levels of oxidative stress. The long-term results of the induced chronic state include accelerated cellular aging.

Ryder, Azcarate, and Choen (2018) conducted a meta-analysis focused on the long-term consequences of chronic PTSD. They found strong 
evidence for elevated risk of musculoskeletal, metabolic, and cardiovascular conditions among those with the PTSD condition. Earlier, Sumner et al. (2017) explored the association between trauma exposure, chronic PTSD, and biomarkers of inflammation in middle-aged women in the Nurses' Health Study II. Their results revealed an increase in inflammation as well as impaired endothelial function leading to the speculation that the inflammation might be the vehicle through which chronic PTSD may increase cardiovascular disease risk.

There is little remaining doubt that PTSD impacts selective organs in the body. A large-scale consortium study was conducted by the Psychiatric Genomics Consortium (PGC)-Enhancing Neuroimaging Genetics through Meta-Analysis (ENIGMA) PTSD Working Group; the group achieved the most extensive neuroimaging study of PTSD to date analyzing data from 1,868 subjects consisting of both PTSD and control group participants. Eight subcortical structures were subjected to a standardized image analysis and quality-control neuroimaging analysis. The results of the meta-analysis revealed a smaller hippocampus size in those participants with PTSD (Logue et al., 2018).

Finally, a significant step is in place through the development of a comprehensive PTSD Biomarker Database (PTSDD) focused on fluid-based biomarkers. Information was collected from over 100 PTSD-related articles ranging from 1997 to 2017. For inclusion in the database, it was necessary for the article authors to evaluate fluidbased biomarkers in humans comparing PTSD patients to control populations. The goal of the project is to investigate whether single biomarkers such as cortisol may emerge as a systemic indicator from at least 900 physiological markers to assist in the early identification of those who will develop PTSD (Domingo-Fernández et al., 2019).

There appears to be cumulative research producing substantial evidence supporting our perspective that PTSD creates a neuroinflammatory state within the afflicted individual. It is likely that the long-term effects contribute to delayed onset PTSD (Frueh, Grubaugh, Yeager, \& Magruder, 2009) as well as to the differential found between combat veterans and noncombat veterans in regards to the later emergence of dementia in their functioning as they attain an older age (Health, 2010).

\section{Memory Reconsolidation Process}

Before the turn of the century (2000), it was thought that memory was permanently stored in the mind in an unalterable fashion. In truth, the hypothesis of memory consolidation was proposed by Müller and Pilzecker about 100 years ago, as cited in McGaugh (2000). The first time a memory is permanently stored in the brain, it is referred to as consolidation. The stronger the memory, such as that which occurs in a traumatic event, the more robust the storage aspect will be.

After a trauma encounter, each time the memory is brought into awareness, it then goes through a reconsolidation process. We have found that such a natural occurrence provides us with an opportunity to alter what is to be restored and what emotional aspect may be dropped out. Thus, parts of the memory become briefly vulnerable to change during the period before it reconsolidates or hardens into long-term memory again. The primary brain region associated with the reconsolidation process is the hippocampus (Dudai, Karni, \& Born, 2015). Our specific goal in RESET Therapy is to sustain the memory detail but erase the negative and traumatic part of it so that it no longer adversely triggers the patient. The individual still has the memory, but it becomes something diminished in the distance and will no longer be experienced as intrusive or disrupting.

We consider RESET Therapy to be a transformative approach wherein specified emotional aspects of the traumatic memory are diminished or erased from long-term memory. The extrinsic memory of the event remains, but the intrinsic emotional aspect has dropped out once the memory has reconsolidated (Ecker, Ticic, \& Hulley, 2012). We find that trauma remediation is possible through brief disruption of the memory reconsolidation process by a neuromodulated sound tuned in to resonate and target with a particular trauma frequency (Lindenfeld \& Bruursema, 2015).

As elucidated by Ecker et al. (2012), we have based our understanding of their working model of memory reconsolidation related to their transformative memory reconsolidation principles. We have found that when selectively tuned in and a binaural sound is correctly applied to maladaptive cortical circuitry, disruption of memory reconsolidation occurs that permits the brain to return to homeostatic norms. We believe that our contribution to a working model of brain plasticity allows the fine-tuning of new protocols for the treatment of PTSD and other treatment-resistant conditions (Lindenfeld \& Rozelle, 
2015). Added to the use of a binaural sound protocol is the conceptual model of neuronal networks and how emotionally related circuits alter the effects of trauma on the memory reconsolidation process (Falconer et al., 2008).

Other therapies are being applied based on the same model, such as the use of Propranolol for disrupting memory reconsolidation of PTSD symptoms (Giustino, Fitzgerald, \& Maren, 2016). An example of a verbally based therapy using transformative principles is Rapid Resolution Therapy (RRT; Harnes, 2010). Within the RRT approach, the therapist seeks to emotionally elicit a traumatic experience while concurrently creating a positive experience, thereby resulting in a mismatch phenomenon.

Changes produced through memory reconsolidation interventions are long-lasting as noted by Monfils, Cowansage, Klann, and LeDoux (2009). Additionally, Beckers and Kindt (2017) noted that “... a recurring theme will be how further basic research and theoretical progress regarding the nature of emotional memory and its modification will inform the future of memory reconsolidation interference as an effective intervention for emotional disorders" ( $p$. 2).

We find that RESET Therapy accomplishes the above-stated objectives without the necessity of drug usage with its accompanying adverse side effects (American Addiction Centers, 2019). Also, because the intervention is nonverbal, it protects the practitioner from exposure to raw limbic system emotional material, thereby protecting the therapist from the secondary effects of PTSD (Penix, Kim, Wilk, \& Adler, 2019).

Our review of the use of binaural sound or the use of any sound for the remediation of PTSD symptomology has found a sparsity of positive results. Alternatively, the literature is replete with instances of sound used primarily as a noxious stimulus (Golkar, Tjaden, \& Kindt, 2017; Schroyens, Beckers, \& Kindt, 2017). We have found that there exists a functional neuroanatomical basis for the proposed therapeutic process (Miller \& Lindenfeld, 2017).

In contrast, rather extensive recent research efforts are related to varied applications of binaural sound such as allocating attention (Colzato, Barone, Sellaro, \& Hommel, 2017); enhancing long-term memory (Garcia-Argibay, Santed, \& Reales, 2017); synchronization of working memory (Beauchene,
Abaid, Moran, Diana, \& Leonessa, 2017); assisting older patients with depression (Sung et al., 2017); patients undergoing general anesthesia (Flanagan \& Kerin, 2017); Parkinson's Disease (Gálvez, Recuero, Canuet, \& Del-Pozo, 2017); relapse prevention (Sewak \& Spielholz, 2018); and reducing preoperative anxiety in dentistry (Isik, Esen, Büyükerkmen, Kilinç, \& Menziletoglu, 2017).

\section{Therapeutic Strategies}

The current use of counteractive procedures in the treatment of PTSD includes extinction (Helpman et al., 2016) and cognitive-behavioral strategies (Monson \& Shnaider, 2014). These procedures are designed to prevent symptom arousal by arranging for a more desired state of mind to emerge as an outcome; similar interventions would include the teaching of relaxation skills to counteract anxiety (Dahlgaard et al., 2019). Another therapeutic strategy would include the cultivation of positive rational thoughts to counter automatic depressive or anxious ones (Agbu, 2015).

\section{Extinction Methods}

Extinction methods are suppressive (Dunsmoor, Niv, Daw, \& Phelps, 2015). They compete against unwanted learning by building up preferred learning and reactions intended to override and suppress the adverse response (Gieselmann et al., 2019). Furthermore, extinction is imperfect, and while some emotional responses may weaken and discontinue, other less desirable emotions are evoked or triggered due to unforeseen circumstances (Pfitzer, 2008). The suggestion here is that deletion or elimination of the original implicit memory did not occur as intended. Maren and Holmes (2016) and Widholm (2010) found that extinction methods have a very high relapse rate in the treatment of addiction indicating that, while the neuronal circuits may become dormant for new learning, they retain their sensitized or potentiated state; once reactivated, old urges (memories) emerge with their former strength.

\section{Biofeedback and Neurofeedback-based Interventions for PTSD}

Biofeedback and neurofeedback are client-centered and empirical data-driven approaches. Feedback informs and empowers the client. Transparency of the procedures allows skeptical patients to observe for themselves what is happening in their brain and body during a session, and to evaluate the trustworthiness of the process and the practitioner quickly. 
Biofeedback researchers established an association between chronic sympathetic hyperarousal from posttraumatic stress and cardiovascular disease risk (Buckley \& Kaloupek, 2001; Kibler, 2009), especially among veterans diagnosed with PTSD (Orr et al., 2000; Orr, Meyerhoff, Edwards, \& Pitman, 1998). Persistently elevated heart rate and attenuated heart rate variability in response to chronic and situational stress was predictive of postdeployment PTSD (Nagpal, Gleichauf, \& Ginsberg, 2013; Chalmers, Quintana, Abbott, \& Kemp, 2014; Dennis et al., 2016; Pyne et al., 2016).

Such alarming findings prompted biofeedback scientists and practitioners to begin using heart rate variability coherence training among traumatized veterans to help them learn balancing of sympathetic and parasympathetic activity (Lake, 2015; Lande, Williams, Francis, Gragnani, \& Morin, 2010; Tan, Dao, Farmer, Sutherland, \& Gervitz, 2011; Tan, Wang, \& Ginsberg, 2013; Wahbeh \& Oken, 2013; White et al., 2017). Breathing retraining techniques were incorporated as a treatment adjunct to aid in the reduction of PTSD symptoms (Polak, Witteveen, Denys, \& Olff, 2015).

Despite the very promising findings of various individual studies, biofeedback was not recommended for management of posttraumatic stress in the VA or Department of Defense (DOD; Psychological Health Center of Excellence, 2018), having failed to meet the "burden of evidence" required by most VA/DOD publications. A seminal study published by Peniston and Kulkosky (1989) demonstrated that a combination of biofeedback and neurofeedback gives veterans a substantially better outcome than treatment-as-usual in a residential addiction treatment setting. Working with veterans at a VA facility in Colorado, Peniston and Kulkosky used techniques developed by Elmer Greene (Greene \& Greene, 1977) to access luminal states of awareness. In addition to treatment-as-usual such as 12-step programs, individual psychotherapy, medication management, and milieu therapy, an experimental group received biofeedback training which included hand-warming, autogenic exercises, and progressive muscle relaxation to lower autonomic arousal. They then underwent 30 consecutive daily individual sessions of alpha-theta neurofeedback where reinforcing amplitude increases in alpha and theta and inhibiting subconscious states where memories and associated emotions are stored, bringing them to conscious awareness. The results were remarkable. Compared with treatment-as-usual controls, veterans in the experimental condition had abstinence rates as high as $70 \%$, and these results were maintained at a follow-up.

Peniston and Kulkosky (1991) turned their attention to Vietnam veterans with combat PTSD. They recruited 29 Vietnam veterans suffering from chronic war-related PTSD, including frequent anxietyevoking nightmares and flashbacks. In comparison to 14 control group participants who received psychotropic medications including tricyclic antidepressants, antipsychotics, and anxiolytics, the 15 experimental group participants received biofeedback training to reduce sympathetic arousal, followed by 30 sessions of alpha-theta neurofeedback, each session lasting $30 \mathrm{~min}$. Some veterans experienced strong emotional reactions during the alpha-theta sessions, supported and encouraged through the session by Dr. Kulkosky. By the end of treatment, outcome measures showed significant symptom reduction, consistent with patient reports. At a 30-month follow-up, all the control participants who received medication treatment alone had experienced a recurrence of PTSD symptoms, whereas only $20 \%$ (3 of 15 ) of the experimental participants reported a recurrence of PTSD symptoms.

The alpha-theta protocol was tested in dually diagnosed veterans having PTS with addiction by Peniston, Marrinan, Deming, and Kulkosky (1993). Peniston et al. (1993) reported that alpha-theta brainwave training significantly reduced anxietyprovoking nightmares, flashbacks, cravings, and urges. Only $20 \%$ of veterans (4 of 20 ) who underwent the alpha-theta protocol reported relapse of alcohol or substance use. Many participants reported that traumatic recollections no longer elicited anxiety. The alpha-theta protocol has since been well replicated in several residential addictions' programs throughout North America (Moore et al., 2000).

In the millennium, researchers continue to replicate Peniston and Kulkosky's basic findings with slight modifications in methodology in veteran and military samples. As part of a military-related stress reduction program, Putnam (2000) showed a relaxation induction video followed by one-channel alpha and low beta amplitude enhancement training at site $\mathrm{Pz}$ using visual feedback in a sample of 77 Army reservists. He found that increases in alpha amplitude eyes-open training was associated with reports of increases in energy level, positive mood, reduced arousal, and, in some cases, reduced vigilance. 
In a doctoral dissertation, Smith (2008) studied 10 military veterans with PTSD-induced depression and decreased levels of attention. After 30 sessions of neurofeedback alpha-theta training, all participants showed a significant reduction in PTSD and depression symptoms and an increase in attention levels. In the aftermath of Operations Iraqi Freedom and Enduring Freedom, mental health researchers in the middle east have replicated the Alpha-Theta protocol with traumatized soldiers (Noohi, Miraghaie, Arabi, \& Nooripour, 2017; Rastegar, Dolatshahi, \& Dogahe, 2016).

Using low-resolution electromagnetic tomography (LORETA) $z$-score training, Foster and Thatcher (2015) reported on the results of the first 16 veterans from an ongoing study of combat veterans with comorbid PTSD and mTBI. Training multiple neural networks implicated in PTS, they reported normalization of the affected networks together with rapid resolution of PTSD symptoms, using protocols customized to the individual aspects and needs of the veteran.

These studies suggest that, regardless of the specific method used, positive outcomes in PTSD are likely using a combination of biofeedback and neurofeedback-each approach complementing the other. The primary limitations of biofeedback and neurofeedback are cost and time. The cost of training, equipment, staff, and malpractice insurance can be daunting. Most insurance plans do not yet recognize neurofeedback as a cost-effective intervention; most patients must pay out of pocket for services. Despite the invaluable information that they provide, qEEG brain maps are relatively expensive, and it may not be practical to use qEEG or neurofeedback as a first-tier treatment approach.

Even when delivered by an experienced and highly skilled practitioner, a minimum of 10 to 20 neurofeedback sessions are necessary before the client consistently experiences positive changes. All neurofeedback practitioners have to deal with the frustrating reality of no-shows and dropouts. The practitioner is under a great deal of pressure to deliver results rapidly, lest impatient clients give up prematurely because progress is taking too long and perceived as too costly. The problem is further magnified in the case of the veteran with PTSD or combined PTSD/TBI with low frustration tolerance, impulsivity, avoidance tendencies, and a high threshold for developing trust.

Rapid remediation of combat-incurred PTSD reengages the prefrontal cortex the center for reasoning, planning, and understanding and establishes the essential trust that motivates the veteran to continue in their biofeedback and neurofeedback treatments. Suffice it to say that following remediation of PTSD symptoms, the veteran's brain still needs a rebalancing of normal asymmetry and stabilization/regulation of thalamocortical, limbic, and brainstem circuits. The veteran's autonomic nervous system must be rebalanced in terms of healthy levels of sympathetic versus parasympathetic arousal. He or she needs to learn readily-usable strategies for managing bodily reactions to stress and sustaining therapeutic gains. Integration of the remediated traumatic memory into one's personal identity to derive new meaning allows a new sense of purpose and future, as opposed to being stuck in the moment. Biofeedback and neurofeedback have essential roles to play in the long-term therapeutic process.

\section{BAUD Biofeedback Device}

In line with the above objective, we have adopted the Bio-Acoustical Utilization Device (BAUD) as the vehicle through which to provide the binaural sound effect (Lawlis, 2006). The BAUD is a handheld, battery-powered device and was FDA approved as a class 2 accessory medical device (Biofeedback device 21 CFR 882.5050) in 2006. The FDA approval is for use in relaxation and stress reduction. The BAUD provides sound through a set of headphones. It has independent volume controls for the left and right ears. It also has a tone (frequency) knob to adjust the sound frequency as well as a "disrupter" adjustment (offset) knob which produces a sound in the left ear that is slightly different than that provided in the right ear. The frequency knob ranges from 30 to $360 \mathrm{~Hz}$ (square wave), heard initially in both ears but later in the right ear. The disruptor knob adds an offset ranging from 0 to $20 \mathrm{~Hz}$, heard in the left ear (see Figure 1).

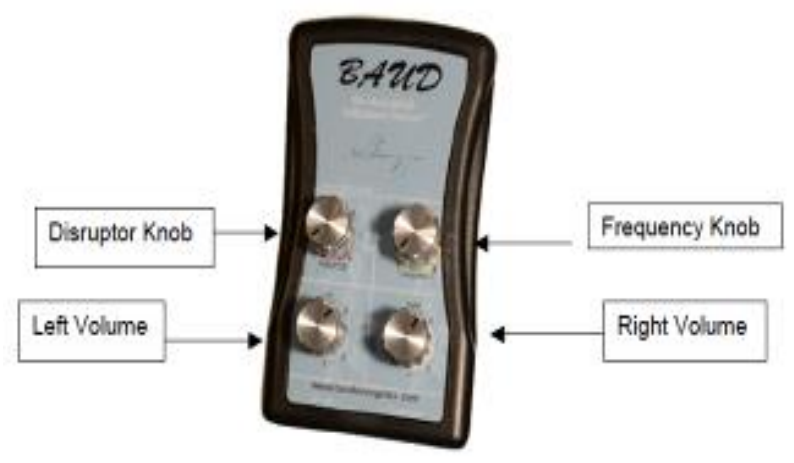

Figure 1. BAUD and varied setting dials. 
BAUD therapy based upon Lawlis's basic principles has been available worldwide, and the BAUD protocol was founded on his perception that:

The underlying acoustical physics entrain the general EEG ranges by creating a third tone from the interference ratios between the two frequencies. It is thus purported to influence brain functioning at the unconscious level and perceived emotional functioning at the conscious level (Lawlis, 2010, p. 3).

"We now know that PTSD is often the result of a physical injury to the brain" (Lawlis, 2011, p. 13-14).

Advances in neuroscience have provided greater clarity related to entrainment and brain damage involvement within the PTSD context. RESET Therapy has integrated these updated findings, consequently leading to our understanding that RESET Therapy is a transformative rather than an entrainment process. Furthermore, while injury to the brain may be concurrent to PTSD among our returning veterans, it is no longer seen as being among the causative factors. Instead, TBI and PTSD are recognized as "signature wounds of war" among those deployed to Afghanistan and Iraq (Tanielian \& Jaycox, 2008). Thus, we have come to perceive of RESET Therapy as a further modification and scientifically-based advancement of Lawlis's foundational work with the BAUD.

\section{RESET Versus BAUD}

RESET Therapy is the treatment process utilizing the BAUD which interferes with targeted trauma memories. Through the specialized use of binaural sound, the intervention blocks the restoration of trauma material after it is selectively lit up in the emotional part of the brain through the patient's intentional focus. We use the term "target" in RESET Therapy and suggest that we are going to turn off the "switch" in the brain that produces the PTSD symptoms (Lindenfeld \& Rozelle, 2015).

\section{Specifics that differentiate RESET from BAUD} Therapy include

(a) Patient preparation based on information related to memory consolidation/reconsolidation factors. The patient is taught that the memory aspect of trauma is the primary problem that perpetuates PTSD, not the traumatic incident itself. Significant recent changes in our understanding of how long-term memory is established and maintained are shared with the treatment participant; (b) Patient's sole focus directed towards activating sensory aspects of traumatic experiences. Coaching is next provided in the identification and targeting of physiological sensations as a primer to the activation of critical emotional circuits;

(c) Targeting varied sensitized brain circuitry beyond trauma circuitry for remediation purposes such as that of unresolved grief;

(d) Informing the participant of a 5-hour vulnerability period following the resetting of cortical circuitry. Therapeutically, the veteran is advised to maintain a stress-reduced environment for the stated period. Through following the prescribed procedure, we accomplish Joseph LeDoux's objective of reshaping memory but without the use of drugs or invasive procedures (Bergstein, 2014).

Brain Map EEG Alterations. We find that when neuronal RESET occurs, survival reactions to trauma based on instinct reassume a secondary position within the context of earlier experienced traumatic events. Support for the "shifting" perspective is present in a recent study of acute stress-related disorders wherein the authors found selective enhancement of threatening variables as well as an apparent decoupling between the dorsolateral prefrontal cortex and the amygdala (Luo et al., 2018). Further support for the position is present in a unique 2017 study that investigated EEG brain map alterations in arousal and reactivity in combat-veterans with PTSD. Bangel, Buschbach, Smit, Mazaheri, and Olff (2017) hypothesized that PTSD-afflicted individuals appear to be highly sensitive to subconscious auditory changes in sound patterns. The authors suggest that PTSD-involved individuals are susceptible due to primary survival mechanisms that place executive functioning in an inferior secondary position.

The above finding supports our perspective of sensitivity to sound and frequency variables that appear to be related to trauma effects that produce a "bottom-up" shift in attention. Some perceive the reaction as being a maladaptive alteration in the neuronal network, which in theory we support. We also view such a shift as being related to survival purposes rather than higher thinking processes (Schmidt, Belopolsky, \& Theeuwes, 2015).

Reptilian Brain. Rosenthal (2015) refers to the Triune Brain model, introduced by neuroscientist Paul D. MacLean, explaining the primitive aspect of the reptilian brain stem which assumes control in traumatic experience(s). 
The reptilian brain takes control, shifting the body into reactive mode. Shutting down all nonessential body and mind processes, the brain stem orchestrates survival mode. During this time the sympathetic nervous system increases stress hormones and prepares the body to fight, flee or freeze...for those 20 percent of trauma survivors who go on to develop symptoms of post-traumatic stress disorder (PTSD) -an unmitigated experience of anxiety related to past trauma-the shift from reactive to responsive mode never occurs. Instead, the reptilian brain, primed to threat and supported by dysregulated activity in significant brain structures, holds the survivor in a constant reactive state (p. 2).

\section{Methods}

Our 54-year-old, three-times-married male, Special Forces veteran (MED) attained 32 years of active service as well as 84 months of combat-involved engagements. He agreed to participate in the case study regarding family violence issues to potentially assist other service members with similar difficulties. MED's primary objective was to alter the deteriorating family situation in which he was currently involved. $\mathrm{He}$ had previously been diagnosed with PTSD, social anxiety disorder, and depressive mood disorder. He was facing felony charges in a state judicial court due to intimate partner violence. Consequent to his legal matters, he was court-ordered to leave home with limited visits established between himself and his two young children. He was to avoid all contact with his wife. The events leading up to his consideration for participation in RESET Therapy included a suicidal near-miss as well as a family violence incident that in his mind was directly linked to his PTSD condition. To protect both his family as well as his identity, details related to these matters will remain protected.

Our investigation protocol was approved by the research ethics committee of The QuietMind Foundation of Pennsylvania which is registered with the U.S. Department of Health and Human Services (DHHS), Office for Human Research Protections (OHRP), IORG 0004684 and IRB 0005585. Following a diagnostic intake with the primary investigator (Lindenfeld), MED underwent the following sequence within the context of his evaluative and treatment process. He provided documentation indicating an honorable discharge from military service. Additionally, he was provided with a consent form for participation and acknowledged that while his identity would be protected, results emanating from his treatment would possibly be used in research articles.

The sequence of his participation for which results are provided included (a) psychometric assessments: Clinician-Administered PTSD Scale for DSM-5 (CAPS-5) and Personal Assessment of Intimacy in Relationship Scale (PAIR); (b) brain mapping in the form of surface qEEG and LORETA anaylsis; (c) four RESET Therapy treatment sessions; (d) post-treatment reassessment (repeat CAPS-5 and PAIR; repeat qEEG) and debriefing. The present study hypothesis is straightforward. Within four treatment sessions of RESET Therapy, MED will demonstrate (1) significant reduction of PTSD symptoms as indicated in his CAPS- 5 scores, and (2) qEEG changes that reflect improvement toward normalization.

\section{Psychometric Assessments}

Clinician-administered PTSD Scale for DSM-5 (CAPS-5). The CAPS-5 is the gold standard in PTSD assessment used by clinicians and clinical researchers at both the VA and DOD who have a working knowledge of PTSD. The full interview takes 45 to 60 minutes to assess 20 DSM-5 PTSD symptoms. The CAPS- 5 requires the identification of a single index trauma to serve as the basis for symptom inquiry. The selected trauma is also utilized within the treatment context to serve as the initial target for frequency and binaural sound offset setting. The questions in the scale target the onset and duration of symptoms, subjective distress, impact of symptoms on occupational and social and functioning, improvement in symptoms since a previous administration, overall response validity, and overall PTSD severity. For each symptom, standardized questions and probes are provided. Scores on the instrument are based on a possible total of 80 points (Weathers et al., 2013). Cutoff scores between 31 and 33 are considered to be optimal for the effective diagnosis of PTSD (Bovin et al., 2016). Scores are derived by combining the frequency and intensity of a particular item into a single severity rating. Each item in the scale is combined to represent intensity scores ranging from 0 (Absent) to 4 (Extreme). A severity score at 2 or above on a particular item suggests clinical significance.

Personal Assessment of Intimacy in Relationship Scale (PAIR). Schaefer and Olsen (1981) created the Personal Assessment of Intimacy Scale (PAIR). The primary focus of the scale is to describe the partner relationship regarding how each currently perceives it. Permission was sought from the 
authors and approved for the use of the scale to determine pretreatment versus posttreatment changes in a specified individual related to intimacy issues.

The five types of intimacy utilized in the above scales are (a) Emotional Intimacy, experiencing a closeness of feeling with others; (b) Social Intimacy, having common trends and similarities in social networks; (c) Intellectual Intimacy, the experience of sharing ideas; (d) Sexual Intimacy, the experience of sharing general affection and/or sexual activity; and (e) Recreational Intimacy, the sharing experience of interest in hobbies such as mutual participation in sporting events. The PAIR instrument does not have cutoff scores, although average scores are expected to be around the mean. The higher the score on each variable, the more likely that a positive outcome on that variable will be more forthcoming (Schaefer \& Olson, 1981).

\section{Brain Mapping}

Surface qEEG. A qEEG is a painless and relatively noninvasive experience. A cap with 19 electrodes is placed on the scalp with two additional linked references placed on the earlobes. A conductive paste is inserted into 19 openings in the cap to ensure a proper connection with the scalp. Each electrode records brain electrical activity in a different region of the scalp, which is a good approximation of activity in the underlying neocortex. The patient is asked to follow standard procedures such as to sit quietly and still, with eyes closed or open, and to reduce eye movement. For RESET cases, the patient may be asked to imagine an emotionally disturbing triggering event during the recording.

The results of the spectral analysis are displayed in color-coded topographic maps. The reported qEEG was based upon at least 60 seconds of edited raw EEG data for each testing condition. Analysis of edited raw EEG data is matched for age, gender, and handedness (Kaiser, 2006). qEEG brain electrical activity was recorded with a 19-channel Electro-Cap (Electro-Cap International, Inc., Eaton, $\mathrm{OH})$, using the 10-20 International Electrode Placement System referenced to linked ears, on a Brainmaster Discovery 24E amplifier (BrainMaster Technologies, Inc., Bedford, OH) with the DC Offset reduced to less than 30 millivolts. The sampling rate was 256 samples per second. No activation procedures were used. The raw recording was digitized for data storage and analysis and later manually edited to reduce artifact (eye movement,
EMG, body movement, etc.) and subjected to quantitative spectral analysis.

For purposes of analysis, the raw data were imported into NeuroGuide Deluxe software (Applied Neuroscience, Inc., Largo, FL). Signal amplification and processing were accomplished with an eyesclosed resting EEG (default state) recorded for $5 \mathrm{~min}$ as a pretreatment baseline. Next, with eyes closed, the veteran was asked to focus attention upon the selected combat-related trauma utilized as a target for the CAPS- 5 interview. The recommended state replicates the "activate the target" component of the RESET procedure. Hence, an eyes-closed baseline (default state) and an eyes-closed trigger condition (trauma network activation) were obtained for utilization as a pretreatment baseline.

\section{Low-Resolution Brain Electromagnetic Tomography (LORETA). Pascual-Marqui et al.} (2011) published reliable research support about a new mathematically based method of analyzing electric neuronal activity (EEG) distributions. Lowresolution brain electromagnetic tomography (LORETA) uses a mathematical inverse solution to determine the relative activity of various regions in the brain using surface electrodes (Thatcher, North, \& Biver, 2005). In contrast to surface qEEG, the LORETA method estimates current source densities at deeper cortical levels using 19 channels of EEG data. Advanced source-correlation software is utilized permitting deep brain structures to become visible in the form of a 3D display (Thatcher, 2011). The validation of the procedure permits functional localization, but, more importantly, it provides the methodology to assess dynamic functional connectivity.

Todder et al. (2012) sought to identify and differentiate changes in brain structure between those with PTSD from those without the condition. Statistically significant findings in the theta band range $(4-7 \mathrm{~Hz})$ thought to represent the activity of the limbic system was noted. Specific regions of the brain identified among the PTSD group as compared to the non-PTSD group included the right and left frontal lobes regions as well as the right temporal lobe.

Cannon et al. (2012) ascertained the validity and reliability of the qEEG over 30 days. Results were good to very good with comparative LORETA calculations at $1 \mathrm{~Hz}$. Across time reveal robust similarity. Data analysis found good to excellent reproducibility of qEEG measures and LORETA. Cannon, Lubar, Thornton, Wilson, and Congeto 
(2004) utilized LORETA to explore the effects of anger in the limbic region of the brain as well as to identify the primary EEG frequencies produced by emotional activation. The participants were asked to elicit memories of anger and to maintain it during the measurement period. Significant differences between the baseline and anger conditions in both frontal and limbic regions supported the perspective that effective memory recollection could be captured for comparative purposes between varied PTSD and non-PTSD groups. An interesting difference observed was that in some cases, the amygdala and uncinate gyrus was activated by beta frequencies $(12-32 \mathrm{~Hz})$. The identified hemispheric asymmetries produced by the above research lend support related to the apparent lateralization of hemispheric activity during to affective reactivity.

\section{RESET Therapy Protocol}

The RESET Therapy protocol is copyright-protected and, as stated, utilizes the commercially available BAUD instrument for producing binaural beats. Those practitioners who perform RESET must be appropriately trained. Although the protocol is described in sufficient detail to allow independent replication, there are nuances as well as caveats that can be learned only by hands-on instruction, observation, and practicum. That training is currently available through the RESET Therapy Professional Institute, LLC.

Guidelines. Before starting each session of RESET:

(1) Dispense with the words Think and Feel. Remove them from your vocabulary. These words are evaluative and will engage the left hemisphere. Our goal is, as much as possible, to minimize the involvement of the left hemisphere, and shift into right-brain experiential processing (sensory focus on pictures, sounds, odors, tastes, and bodily sensations). The right brain can respond with single words (e.g., Yes, No) without much conscious evaluation.

(2) Use the words You and I. Dispense with the words We or Us.

(3) Use the patient's own words (descriptions of sensations) rather than your words. The meaning of words can be easily misconstrued, so it is best to reiterate their words (e.g. the flicker/tightness in your chest, the lump in your throat, etc.)
(4) Closely observe the patient's behavior for signs of shifts in arousal, both during the tuningin phases and during the actual exposure trials.

(5) Keep talking to a minimum.

(6) When first working with a new patient, choose a memory with a Subjective Unit of Distress Scale (SUDS; Kaplan, Smith, \& Coons, 1995) rating of about a 6 or 7 , based on a 10point range (10 most intense) rather than a higher SUDS memory.

(7) Before doing your first RESET session, get familiar with the technique of ideomotor signaling. Ideomotor signaling was effectively used in medical hypnosis by LeCron (1964). It is a form of nonverbal communication, akin to nodding the head Yes, turning the head from side to side No, or shrugging the shoulders with I don't know. Patients are taught how to allow the subconscious mind to signal a response, while the patient is simultaneously and intensely focusing attention upon his or her subjective sensory/bodily experiencing and the sound of the tone.

(8) Phase 1 of the RESET assessment phase involves having the patient activate or "light up the target" from the present adult perspective (looking back at the disturbing memory). We can anticipate that the patient will start in a relaxed state with eyes closed (alpha) but will rapidly shift to a state of negative arousal once the bodily sensations resonate with the BAUD frequency tone being adjusted by the therapist.

(9) Phase 2 of the RESET assessment phase invites the patient's subconscious mind to become involved by engaging a child-like perspective. The dominant EEG rhythm in children is theta, which is associated with daydreaming, fantasy, and creativity. Theta is also the frequency in the adult where subconscious memory and emotion can be most readily accessed. We seek to engage subcortical areas as well as the right cerebral hemisphere, to process sensory and perceptual experience associated with the memory of the traumatic event(s). The subconscious mind is very literal and concrete, child-like in many aspects. When we fine-tune the BAUD settings while the client is accessing an unembellished state of awareness, we obtain the best results. 
(10) If the patient is having difficulty zeroing in a key memory, ask him or her to remember back to a time when things were "normal" or trouble free. Then ask at what point things began to change (a specific stressor or series of stressors). Hence, ask the patient to go back chronologically through the emergence of the trauma. If the patient is dissociated (has difficulty identifying bodily sensations), use a lower intensity target. If dissociative, the therapist may have to do a couple of Eye Movement Desensitization and Reprocessing (EMDR) sessions first.

\section{RESET Therapy with MED}

Clarification was provided in detail to MED that it was necessary to light up the target in the emotional region (limbic system) of the brain. He was informed that the sensory aspects of a selected trauma experience need to be activated rather than merely thinking about it. The veteran was told that the best way to create the experience was to imagine being in the experience fully and entirely by bringing in all the involved senses and thoughts that were present at the time of the experience, including sight, sound, smell, and skin sensation.

An explanation was provided about the SUDS, utilized in the study ranged from a level of 0 to 10 , to determine the subjective level of intensity of the targeted trauma. MED identified an intensity score at the most exhaustive point during the period of imagery and then again following the treatment intervention. The therapist observed and monitored physiological reactivity, including facial expression, respiration rate, and muscle tension.

Treatment Summary. MED completed his treatment involvement following three RESET Therapy sessions perceiving that all his traumarelated symptoms remediated. $\mathrm{He}$ was consequently able to provide clarity related to many associated triggers that had considerably weakened his ability to contain his rage and anger. $\mathrm{He}$ described these triggers as follows:

[The first event was when] I conducted a battlefield assessment for what was known as the "Highway of Death"-Highway 80 between Kuwait and Iraq. During that event, I witnessed burnt and charred bodies in different states of decay that left a lasting negative impression on me. I lost my faith in religion, and, quite frankly, I lost a lot of faith in mankind.
The second event occurred on the job in Iraq in 2007 where we did a "sensitive site exploitation" related to an attack by around 500 insurgents on a special forces team where an estimated 350 to 400 attackers were killed. The amount of destruction put on those individuals was rather grotesque. Large caliber Gatlin Gun bullets and explosive bombs/missiles were used that pretty much ripped those guys apart. The two events were similar. The only difference was that the bodies were much fresher than the ones l'd seen earlier.

The third event was the loss of a good friend during the invasion of Afghanistan. I went through the Special Forces selection with him, including the Forces qualification course. We went to the same Special Forces group and maintained a close relationship. His loss hit me pretty hard, and it was one of those things that stuck with me. Not being able to see him again, hear him and wishing I could be with him. The level of friendship between us was very close. It was a significant loss.

In his first 5-min trial RESET Therapy treatment, MED recalled that when the sound frequency reached a certain level:

My body tensed up to a point where it felt like a muscle spasm. One-third of the way through it, I had an internal dialogue-stop trying to control everything. I experienced a pulsing from inside to the back of my head and then tingling through the rest of my body. My right leg jumped a couple of times. At first, I rated the level at a 6/7 on a 10-point scale. After the 5-min trial, it was at a 0 . I can't bring it up right now (MED, personal communication, March 15, 2018).

Before his second treatment, MED shared that he usually tries to avoid trigger situations:

I was curious about how the treatment took, so I tested it at home with stuff from Desert Storm and Iraq. I imagined seeing dead guys. Also, the smell of barbeque would trigger me. I couldn't put myself into it. My sleep was different because I got a good and deep rest. A court veteran employee who also went through the program testified, and the judge reduced my charges to a misdemeanor. I was also able to move back into the home because my wife testified that I was no longer a danger to her. I'm now back in the house with my wife and kids 
(MED, personal communication, March 27, 2018).

At his third and final treatment visit, MED reported that his wife was no longer drinking, and he was feeling great. He was told to focus on the loss of his close friend in a treatment tweak that I have called, "talking to your dead buddies(s)." He reported the following:

When Doc asked me to be with my dead buddies, in my mind, I thought, "oh great, I'm going to be a seance kind of thing." I thought, "you know what-l'll try anything at this point." It turned into a unique experience. I started in the aftermath of the event of his loss. I went into sadness at what it meant, but then transitioned into a conversation with my buddy. He's funny, and he laughed about his death. If he could laugh about his death, then I shouldn't be so sad. He told me he's in a good place. I hugged him. Seeing his face again, I could feel happy tears on my face. I let go of a lot of suppressed emotion. I don't feel I have to lock everything in anymore because l've released the poison. I'm now at a 0 on the skepticism scale.

By the time we finished the third treatment, I had decided that Doc George was on to something. There's no question that my life has finally changed, all for the better. P.S. I completed my homework assignment with flying colors by watching "Twelve Strong," a film about Special Forces from my time. I actually enjoyed it! (MED, personal communication, April 16, 2018).

\section{Results}

\section{CAPS-5 Results}

MED's pretreatment CAPS- 5 score of 58 , as seen in Figure 2, was well within the range expected for those who are diagnosed with PTSD. In contrast, his posttreatment CAPS-5 scores dropped significantly to 5 following three 20-min RESET
Therapy treatments. Following his brief treatment, he no longer met the criteria for the PTSD diagnosis. We may thus assume that his trauma-initiated behavior has reverted (remediated) to a homeostatic level permitting a full range of effective expression as compared to a prior restricted range based primarily on survival needs.

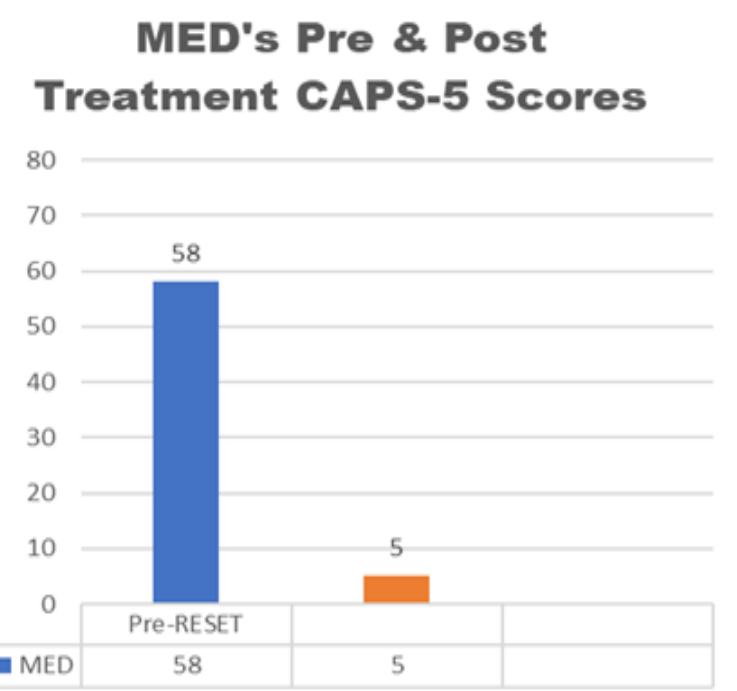

Figure 2. MED's Pre- and posttreatment CAPS-5 scores. The blue bar represents MED's pretreatment score, while the orange bar is the posttreatment status perspective.

\section{PAIR Results}

MED's PAIR results, as noted in Figure 3, reveal a significant shift in relationship interest and participation, with higher posttreatment scores relative to pretreatment scores, on all measured variables. By far, the most dramatic change is evident on the Sexual variable with a pretreatment score of 20 changing to a posttreatment score of 92 . His transformative changes across many measured variables are supportive of his verbal report of his change in the ability to express positive emotions 


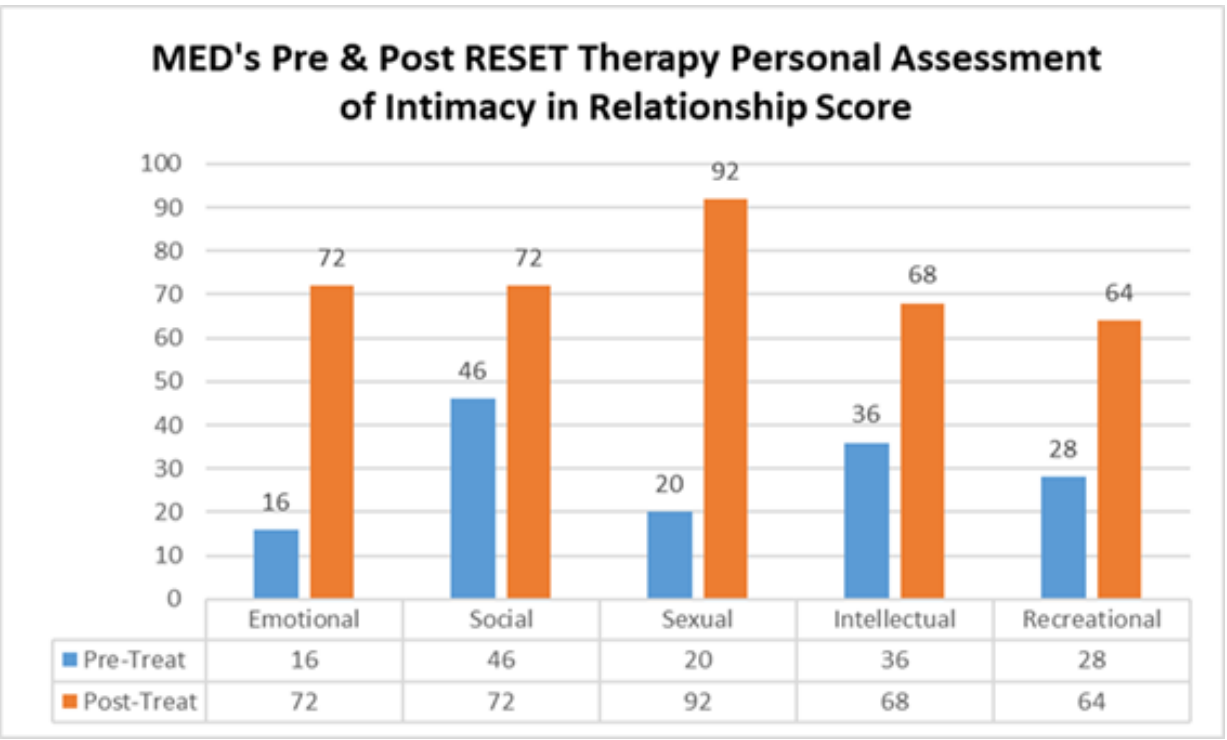

Figure 3. MED's Pre-\& Post Personal Assessment of Intimacy in Relationship Score. The blue bars represent pre-treatment, while the orange bars are post-treatment scores.

\section{Surface qEEG Results}

Resting Baseline. As displayed in Figures 4 through 7, the $z$-score values for the absolute and relative power are represented by a color palette. The green colors represents $\pm 1.0 \quad z$-scores and corresponds to the middle $68 \%$ of the observed population and considered within the average, $z$-score values greater than 1.0 (yellow/orange) or less than -1.0 (light blue) represent dysregulated brain frequencies, and $z$-score values greater than 3.0 (red) or less than -3.0 (dark blue) represent very dysregulated brain frequencies. For asymmetry, coherence, and phase measures, the $z$-score values are represented by red or blue lines, with red indicating above average (i.e., hyper) and blue below average (i.e., hypo) values.

MED's pretreatment eyes-closed resting baseline findings revealed absolute power measures with elevations in delta $(1-4 \mathrm{~Hz})$ and theta $(4-8 \mathrm{~Hz})$ as well as in the bilateral frontal, temporal, and parietal regions. Alpha, beta, and high beta showed left frontal focal dysregulation. Increased delta and theta levels suggested a state of under arousal while at rest.

There was global hypocoherence in all frequency bands, especially in delta, with a corresponding phase lag. These indicators represent a neuronal disconnect suggesting white matter damage. On pretesting, the left side showed the most substantial deviation from normal. The right side showed slowing, but the left overshadowed it. The left side of BA 9 is involved in attention to negative emotion and recall of negative experiences. Posttesting results revealed the left side to have normalized quite well, with the right-side slowing more visible. The finding of improved qEEG following three RESET Therapy sessions suggests that much of his pretreatment condition was likely due to residual trauma effects.

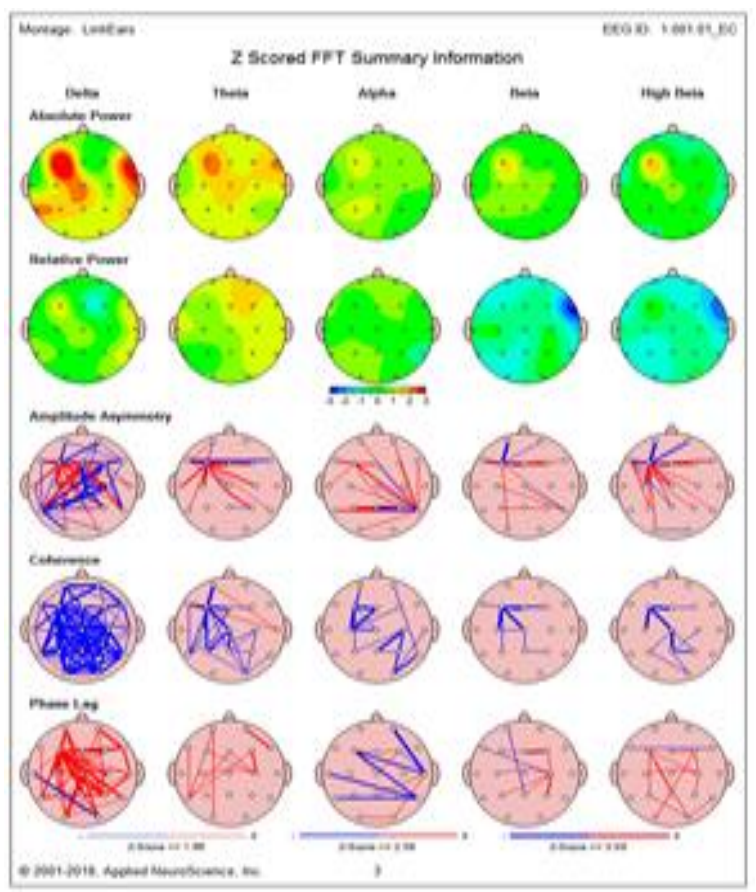

Figure 4. MED eyes-closed resting baseline pretreatment. 


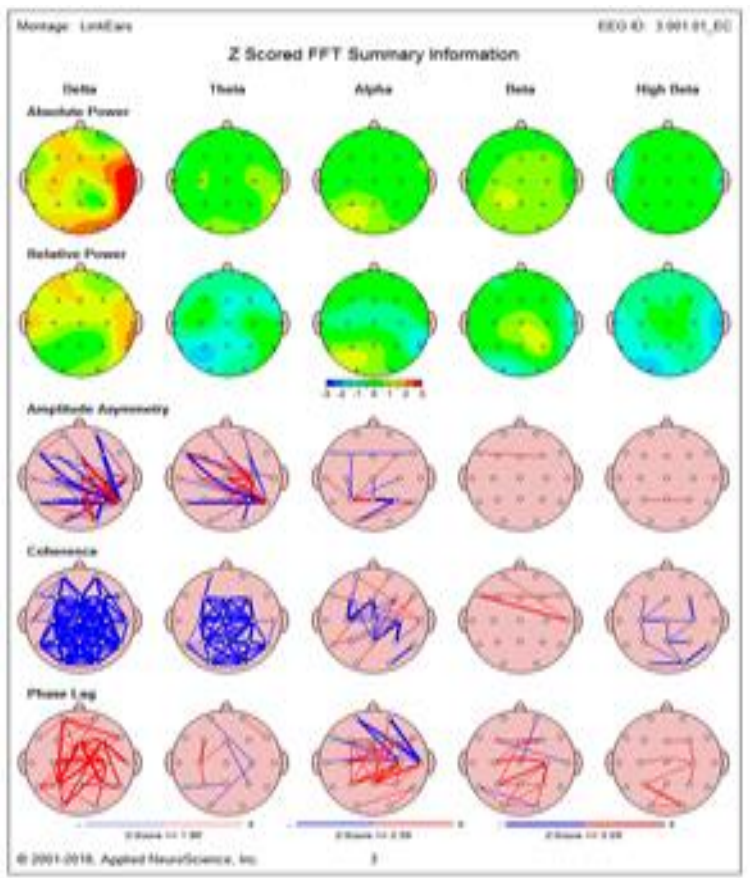

Figure 5. Eyes-closed resting baseline posttreatment.

Trauma Recall: A second 5-min pretreatment recording (Figure 6) was then repeated with MED focused on activating combat-related trauma experiences which presented primarily in the theta range (Dunkley et al., 2015). Elevations that are similar have been found to serve as a protective disengagement mechanism for the veteran. Simultaneously, bilateral frontal lobe dysregulation also was present in the theta pattern suggesting disinhibition of impulse control. Within the assigned task, he visually appeared to be more agitated with increased and shallow thoracic breathing, facial grimacing, and notable muscle tension.

Trauma activation posttreatment qEEG (Figure 7) revealed a significant reduction in the excess delta, and normalization of theta, alpha, beta, and high beta. Coherence and phase were slightly improved. $\mathrm{He}$ visually appeared to be more relaxed with normative breathing evidence. A previously unseen elevation (red area) is evidenced in the delta, right prefrontal region suggesting activation of a region of the brain that engages in impulse control as well as social involvement.

The initial enhancement of activation will likely subside over time as MED adjusts to his new persona. The effect will be further explored within the context of a one-year follow-up re-evaluation. It thus appears that his recorded qEEG changes reveal an atypical yet significant treatment effect. The posttreatment qEEG results provides tentative support to our position that the afflicted PTSD neuronal network has reverted to a pretrauma homeostatic setting.

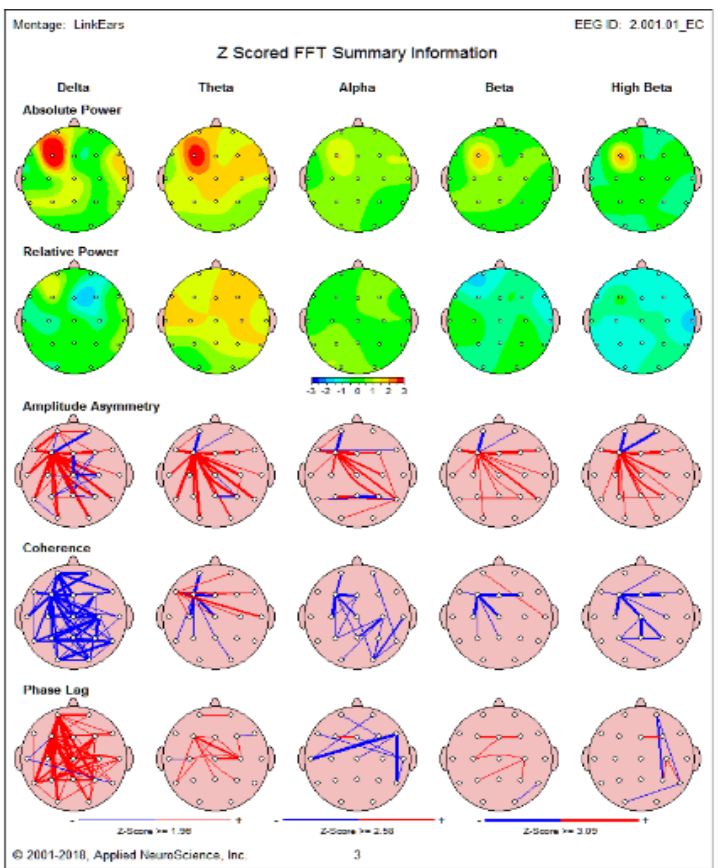

Figure 6. MED trauma recall pretreatment.

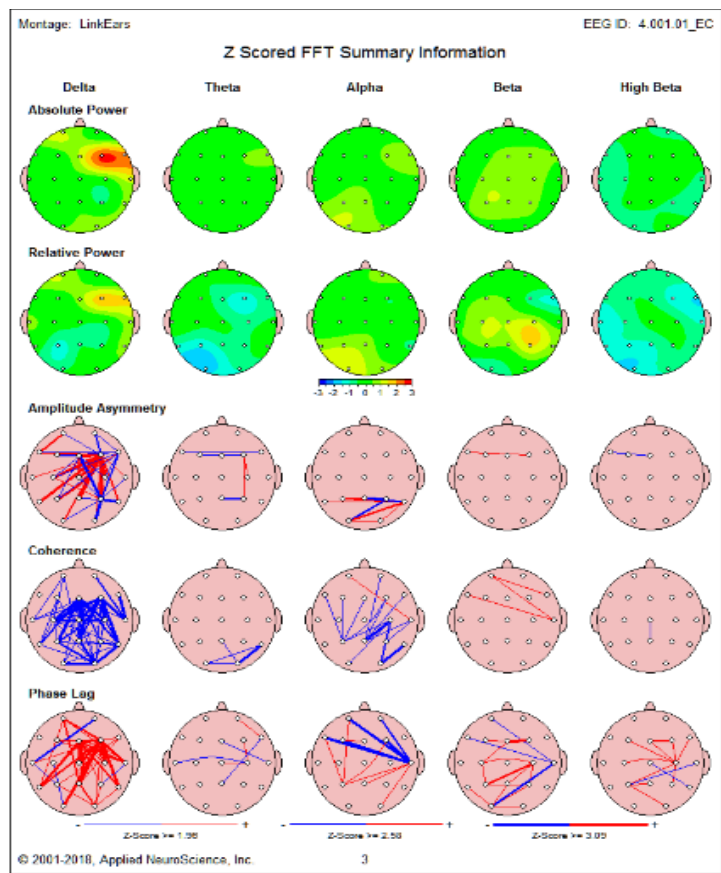

Figure 7. MED trauma recall posttreatment. 


\section{LORETA Results}

As displayed in Figures 8 through 11, LORETA results depict the CSD localization findings and calculated cortical generators as found for the MRI co-registered Talairach coordinates. The red in the image indicates greater CSD amplitude at single hertz frequencies as contrasted to the normative sample.
MED's areas of greatest dysregulation appear to be Brodmann areas (BA); BA 9, 45, resting baseline and in the trigger condition, making it difficult to measure precise changes in these areas when triggered by emotional memories. Frontal lobe dysregulation would result in reduced executive functioning, poor impulse inhibition, depression, and poor sustainment of attention. BA 9 showed the greatest dysregulation in the trigger condition with excess $7 \mathrm{~Hz}$ and $28 \mathrm{~Hz}$, showing the most significant deviations (Figures 8 and 9).

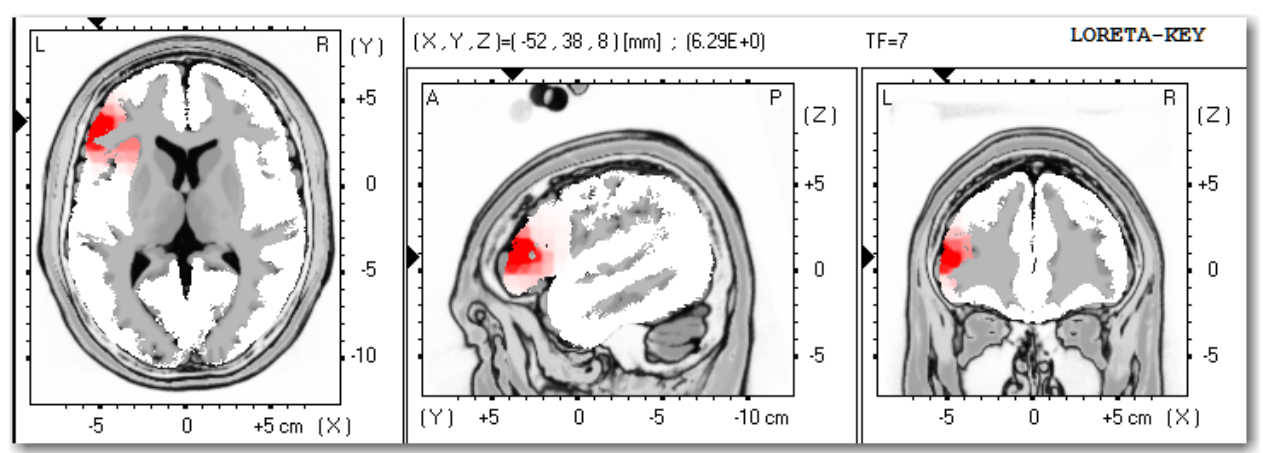

Figure 8. MED trauma recall pretreatment LORETA slice $7 \mathrm{~Hz}$.

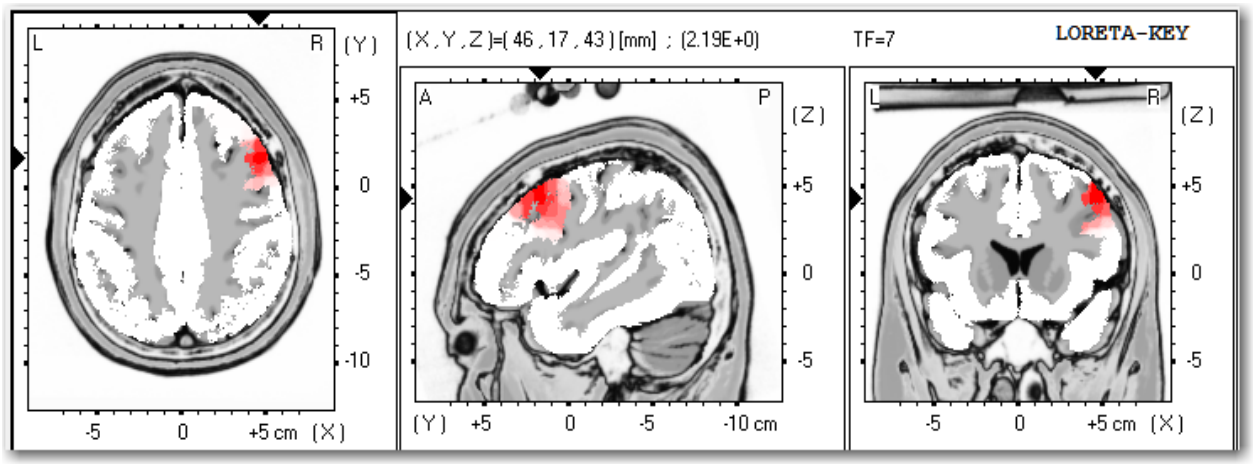

Figure 9. MED trauma recall pretreatment LORETA slice at $28 \mathrm{~Hz}$.

In the posttreatment LORETA data, shown in Figure 10 , continual $7-\mathrm{Hz}$ effects appear present in similar areas including Brodmann area 9, the frontal lobe, and superior frontal gyrus.
In contrast, as evidenced in Figure 11, in the $28-\mathrm{Hz}$ frequency, all indicators are normative. As noted earlier, the LORETA alteration occurred within the context of three RESET Therapy sessions. 


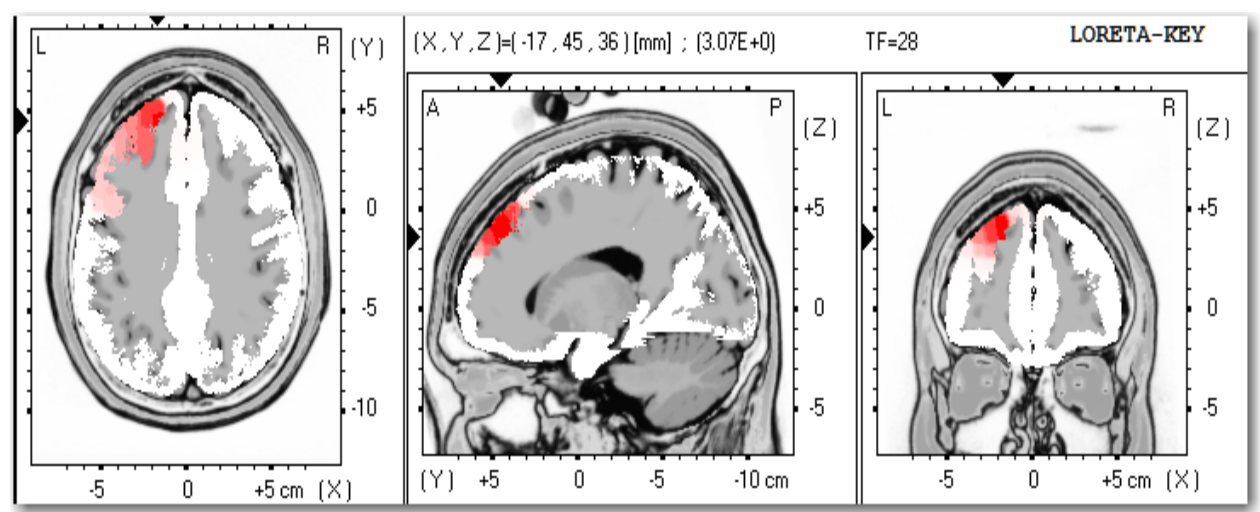

Figure 10. MED trauma recall posttreatment LORETA slice at $7 \mathrm{~Hz}$.

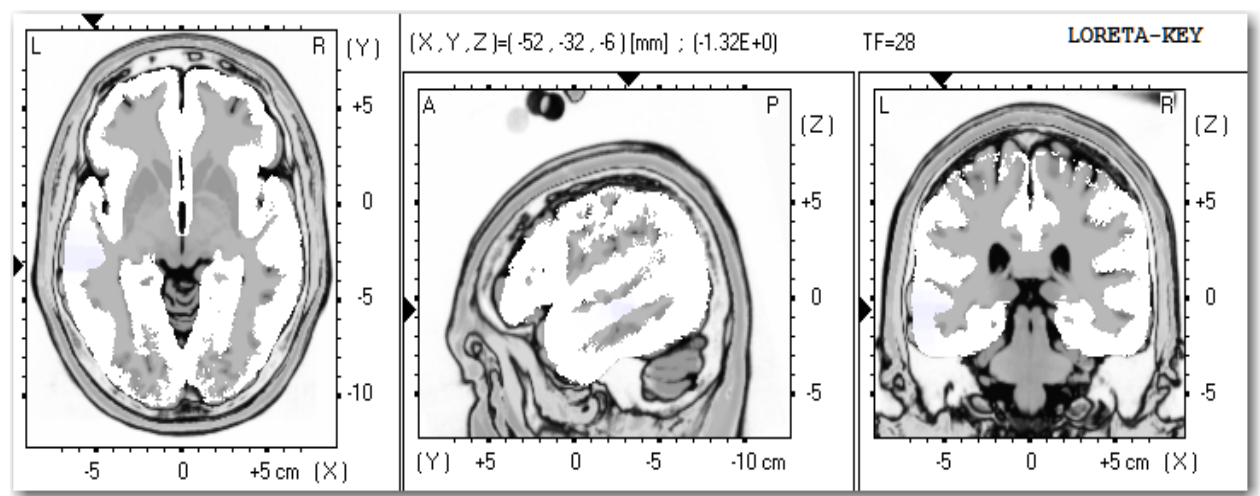

Figure 11. MED trauma recall posttreatment LORETA slice at $28 \mathrm{~Hz}$.

\section{Discussion}

The experiential material later verbalized by the veteran and former Special Forces member certainly makes it understandable how he came to be previously diagnosed with PTSD. MED's pretreatment qEEG also helps us to recognize how he sought to control the rage that was locked within him by cortically disengaging from the unpleasantries that troubled him. Unfortunately, his survival process also served to disinhibit his impulse control abilities. Aside from how MED previously functioned, the changes forthcoming from his involvement with a brief intervention called RESET Therapy appears to have altered his life back to one of relative normality and sanity. We are striving to bring RESET Therapy to the next level-a thorough scientific research study, including a control group to obtain scientific support for this noninvasive, nonverbal transformative therapy.

We believe that the quest to finally end the ongoing nightmare of PTSD for active service members, veterans, first responders, and civilians who are chronically afflicted with the PTS condition is a worthy challenge to pursue. For our case study purpose, we did not solicit a detailed history before treatment other than to establish that there was significant combat-related PTSD that occurred through his military service. The specific content of the PTSD was irrelevant to the RESET Therapy approach as we have come to consider that for many, the nature of PTSD is that the person cannot fully recall details, tries to avoid remembering emotionally charged events, and has trouble fully articulating events.

Based upon previous $\mathrm{FMRI}$ and GEEG studies of PTSD, we hypothesized that brain mapping (surface qEEG \& LORETA) would capture the anticipated changes forthcoming from the RESET Therapy intervention. For example, we postulated that the PTSD condition would include overactivation of the limbic system and underactivation of the medial frontal lobe and Broca's speech area.

We also hypothesized that there would be left and right temporal focal dysregulation present. Baseline measures matched our expectations regarding the neuronal pattern of PTSD hypotheses. We believe 
that silently activating and consequently recalling trauma events assisted in triggering and, therefore, fully capturing the pretreatment brain map findings. It is important to note that pre- and posttreatment qEEGs were recorded with the same imaginal exposure to a specifically referenced combatincurred trauma. Additionally, the data was recorded at the same time of day. As found in Figure 7 (trauma recall posttreatment), MED's brain map revealed significant normalization of the $\mathrm{qEEG}$ in the regions of interest related to PTSD.

\section{Conclusion}

Based on our preliminary findings, we find initial support for our hypothesis related to the systemic aspect of PTSD, apparent neuroinflammatory element, and memory component within the context of the reconsolidation process. For example, the rather dramatic changes seen on the qEEG results, including LORETA, as well as CAPS-5 and PAIR scores seem to substantiate that binaural sound serves as a key to unlock and alter the emotional aspects of long-term memory. Our results appear to be consistent with other transformation-based interventions. While our sample size is limited, the preliminary findings from our unique case study provides objective as well as subjective evidence of change in brain circuitry.

Indeed, sound has been a universal means to energize warriors to enter the fray of battle. Paradoxically, it may also prove to be the means to transform the warrior back to the socialized being that existed before his or her engagement in military life. Sound used for the healing of the soul and body has long existed in numerous cultures throughout time. Perhaps we will soon harness sound for resetting the aberrant circuitry in the brain created by the trauma exposure effect.

While our article is a single qEEG case study, we would hope to explore the RESET phenomenon further and to refine our procedures within the context of a formal research study. The RESET protocol shows initial promise for adding an alternative noninvasive intervention for the treatment of PTSD. Due to the rapidity of response to treatment, we perceive that increased numbers of afflicted individuals could be assisted in shorter periods than our current traditional therapies. Furthermore, due to its nonverbal aspect, it may extend the viability of the trauma therapist's efficiency by shielding the practitioner from the effects of secondary exposure.
From a broader perspective, the neuronal pattern of PTSD appears to be a valid prognostic indicator that captures the varied effects of the PTSD and trauma condition using surface QEEG, LORETA, and psychometric measurements such as the CAPS-5. Further inquiry into the impact that binaural sound may have on the DMN is also a ripe area for further investigation. Finally, utilization of the qEEG serves as validation of change within brain circuitry as well as providing further clarification of the possible presence of underlying traumatic brain injury.

While not intending to overstate our findings, we need to restate the critical need to find interventions that offer the potential to fully remediate those who continue to experience the residual and chronic effects of combat and trauma incurred through their service to our country. We as a country currently lose 20 veterans a day to suicide; it is critical that we do something better to serve those who served us. It is plausible that RESET therapy could be utilized to allow soldiers to recover from trauma-related PTSD and return to work and remain productive soldiers. Those who serve now, and who have served previously, deserve no less.

\section{Limitations}

The results of the provided three RESET Therapy sessions require a final reassessment after a year from the veteran's last treatment date. Tracking MED's ongoing judicial status will provide another way to determine if his interpersonal changes remain intact. Additionally, alcohol abuse within the family context appeared to be an aggravating factor further weakening the husband-wife bond. The above elements require ongoing monitoring over the designated one-year re-evaluation period.

\section{Author Disclosure}

Lindenfeld is the owner of the RESET Therapy Professional Institute, LLC, which is dedicated to the permanent healing of emotional trauma. No financial interest is involved in the sale of equipment (BAUD) utilized as the primary treatment vehicle. The RESET Therapy Professional Institute, LLC has affiliated with the Sarasota Community Foundation (501c3) to seek funding for research purposes. To date, all research efforts have been the results of pro bono contributions of professionals involved. Training leading to certification is in the planning stage for future offerings. None of the other coauthors have financial interests of any kind related to these research efforts. 


\section{References}

Agbu, J.-f. O. (2015). Management of negative self-image using rational emotive and behavioural therapy and assertiveness training. ASEAN Journal of Psychiatry, 16(1), 57-68.

American Addiction Centers. (2019). Post-traumatic stress disorder symptoms, causes and effects. Retrieved from https://www.psychguides.com/guides/post-traumatic-stressdisorder-symptoms-causes-and-effects/

Bam, M., Yang, X., Zumbrun, E. E., Zhong, Y., Zhou, J., Ginsberg, J. P., ... Nagarkatti, M. (2016). Dysregulated immune system networks in war veterans with PTSD is an outcome of altered miRNA expression and DNA methylation. Scientific Reports, 6, 31209. https://doi.org/10.1038 /srep31209

Bangel, K. A., Buschbach, S., Smit, D. J. A., Mazaheri, A., \& Olff, M. (2017). Aberrant brain response after auditory deviance in PTSD compared to trauma controls: An EEG study. Scientific Reports, 7(1), 16596. https://doi.org/10.1038/s41598-01716669-8

Beauchene, C., Abaid, N., Moran, R., Diana, R. A., \& Leonessa, A. (2017). The effect of binaural beats on verbal working memory and cortical connectivity. Journal of Neural Engineering, 14(2), 026014. https://doi.org/10.1088/17412552/aa5d67

Beckers, T., \& Kindt, M. (2017). Memory reconsolidation interference as an emerging treatment for emotional disorders: Strengths, limitations, challenges, and opportunities. Annual Review of Clinical Psychology, 13, 99121. https://doi.org/10.1146/annurev-clinpsy-032816-045209

Bergstein, B. (2014, June 17). The promise and perils of manipulating memory. MIT Technology Review. [Internet Blog]. Retrieved from https://www.technologyreview.com/s /528156/the-promise-and-perils-of-manipulating-memory/

Bovin, M. J., Marx, B. P., Weathers, F. W., Gallagher, M. W., Rodriguez, P., Schnurr, P. P., \& Keane, T. M. (2016). Psychometric properties of the PTSD Checklist for Diagnostic and Statistical Manual of Mental Disorders-Fifth Edition (PCL5) in veterans. Psychological Assessment, 28(11), 13791391. https://doi.org/10.1037/pas0000254

BrainMaster Discovery 24E [Apparatus]. Bedford, $\mathrm{OH}$ : BrainMaster Technologies, Inc.

Brenner, L. A. (2011). Neuropsychological and neuroimaging findings in traumatic brain injury and post-traumatic stress disorder. Dialogues in Clinical Neuroscience, 13(3), 311-323.

Bressler, S. L., \& Menon, V. (2010). Large-scale brain networks in cognition: Emerging methods and principles. Trends in Cognitive Science, 14(6), 277-290. https://doi.org/10.1016 /j.tics.2010.04.004

Buckley, T. C., \& Kaloupek, D. G. (2001). A meta-analytic examination of basal cardiovascular activity in posttraumatic stress disorder. Psychosomatic Medicine, 63(4), 585-594. https://doi.org/10.1097/00006842-200107000-00011

Cannon R. L., Baldwin D. R., Shaw T. L., Diloreto, D. J., Phillips, S. M., Scruggs, A. M., \& Riehl, T. C. (2012). Reliability of Quantitative EEG (qEEG) measures and LORETA current source density at 30 days. Neuroscience Letters, 518(1), 2731. https://doi.org/10.1016/j.neulet.2012.04.035

Cannon, R., Lubar, J., Thornton, K., Wilson, S., \& Congedo, M. (2004). Limbic beta activation and LORETA: Can hippocampal and related limbic activity be recorded and changes visualized using LORETA in an affective memory condition? Journal of Neurotherapy, 8(4). https://doi.org /10.1300/J184v08n04 02

Chalmers, J. A., Quintana, D., Abbott, A., \& Kemp, A. H. (2014). Anxiety disorders are associated with reduced heart rate variability: A meta-analysis. Frontiers in Psychiatry, 11, 80. https://doi.org/10.3389/fpsyt.2014.00080

Colzato, L. S., Barone, H., Sellaro, R., \& Hommel, B. (2017). More attentional focusing through binaural beats: Evidence from the global-local task. Psychological Research, 81(1), 271-277. https://doi.org/10.1007/s00426-015-0727-0

Dahlgaard, J., Jørgensen, M. M., van der Velden, A. M., Sumbundu, A., Gregersen, N., Olsen, R. K., \& Mehlsen, M. Y. (2019). Mindfulness, health, and longevity. In S. I. S. Rattan \& M. Kyriazis (Eds.), The Science of Hormesis in Health and Longevity (pp. 243-255). New York, NY: Academic Press/Elsevier. https://doi.org/10.1016/B978-0-12-814253$0.00022-X$

Davidson, R. J., \& Hugdahl, K. (1996). Brain Asymmetry. Cambridge, MA: The MIT Press.

Dennis, P. A., Dedert, E. A., Van Voorhees, E. E., Watkins, L. L., Hayano, J., Calhoun, P. S., ... Beckham, J. C. (2016). Examining the crux of autonomic dysfunction in posttraumatic stress disorder: Whether chronic or situational distress underlies elevated heart rate and attenuated heart rate variability. Psychosomatic Medicine, 78(7), 805-809. https://doi.org/10.1097/PSY.0000000000000326

DiSabato, D. J., Quan, N., \& Godbout, J. P. (2016). Neuroinflammation: The devil is in the details. Journal of Neurochemistry, 139(52), 136-153. https://doi.org/10.1111 /jnc. 13607

Domingo-Fernandez, D., Provost, A., Kodamullil, A. T., MarinLlao, J., Lasseter, H., Diaz, K., ... Haas, M. (2019). PTSD Biomarker Database: Deep dive meta-database for PTSD biomarkers, visualizations, and analysis tools. BioRxiv, 547901. Advance online publication. https://doi.org/10.1101 1547901

Dudai, Y., Karni, A., \& Born, J. (2015). The consolidation and transformation of memory. Neuron, 88(1), 20-32. https://doi.org/10.1016/j.neuron.2015.09.004

Dunkley, B. T., Sedge, P. A, Doesburg, S. M., Grodecki, R. J., Jetly, R., Shek, P. N., ... Pang, E. W. (2015). Theta, mental flexibility, and post-traumatic stress disorder: Connecting in the parietal cortex. PLOS ONE, 10(4), e0123541. https://doi.org/10.1371/journal.pone.0123541

Dunsmoor, J. E., Niv, Y., Daw, N., \& Phelps, E. A. (2015). Rethinking extinction. Neuron, 88(1), 47-63. https://doi.org /10.1016/j.neuron.2015.09.028

Ecker, B., Ticic, R., \& Hulley, L. (2012). Unlocking the emotional brain: Eliminating symptoms at their roots using memory reconsolidation. New York, NY: Routledge. https://doi.org /10.4324/9780203804377

Electro-Cap system [Apparatus]. Eaton, OH: Electro-Cap International, Inc.

Engdahl, B., Leuthold A. C., Tan, H.-R. M., Lewis S. M. Winskowski, A. M., Dikel, T. N., \& Georgopoulos, A. P. (2010). Post-traumatic stress disorder: A right temporal lobe syndrome? Journal of Neural Engineering, 7(6), 1-8. https://doi.org/10.1088/1741-2560/7/6/066005

Engels, A. S., Heller, W., Mohanty, A., Herrington, J. D., Banich, M. T., Webb, A. G., \& Miller, G. A. (2007). Specificity of regional brain activity in anxiety types during emotion processing. Psychophysiology, 44(3), 352-363. https://doi.org /10.1111/j.1469-8986.2007.00518.x

Falconer, E., Bryant, R., Felmingham, K. L., Kemp, A. H., Gordon, E., Peduto, A., ... Williams, L. M. (2008). The neural networks of inhibitory control in posttraumatic stress disorder. Journal of Psychiatry \& Neuroscience, 33(5), 413-422. http://jpn.ca/vol33-issue5/33-5-413/

Flanagan, D. A., \& Kerin, A. (2017). How is intraoperative music therapy beneficial to adult patients undergoing general anesthesia? A systematic review. Anesthesia EJournal, 5, 513. Retrieved from https://anesthesiaejournal.com/index.php /aej/article/view/62

Foster, D. S., \& Thatcher, R. W. (2015). Surface and LORETA neurofeedback in the treatment of post-traumatic stress disorder and mild traumatic brain injury. In R. W. Thatcher \& J. F. Lubar (Eds.), Z score neurofeedback: Clinical 
applications (pp. 59-92). Waltham, MA: Academic Press. https://doi.org/10.1016/B978-0-12-801291-8.00004-2

Fourrier, C., Singhal, G., \& Baune, B. T. (2019). Neuroinflammation and cognition across psychiatric conditions. CNS Spectrums, 24(1), 4-15. https://doi.org /10.1017/S1092852918001499

Frueh, B. C., Grubaugh, A. L., Yeager, D. E., \& Magruder, K. M. (2009). Delayed-onset post-traumatic stress disorder among war veterans in primary care clinics. The British Journal of Psychiatry, 194(6), 515-520. https://doi.org/10.1192 /bjp.bp.108.054700

Gálvez, G., Recuero, M., Canuet, L., \& Del-Pozo, F. (2017). Short-term effects of binaural beats on EEG power, functional connectivity, cognition, gait, and anxiety in Parkinson's Disease. International Journal of Neural Systems, 28(5), 1750055. https://doi.org/10.1142/S0129065717500551

Garcia-Argibay, M., Santed, M. A., \& Reales, J. M. (2017). Binaural auditory beats affect long-term memory. Psychological Research, 1-33. Advance online publication. https://doi.org/10.1007/s00426-017-0959-2

Gieselmann, A., Aoudia, M. A., Carr, M., Germain, A., Gorzka, R., Holzinger, B., ... Pietrowsky, R. (2019). Aetiology and treatment of nightmare disorder: State of the art and future perspectives. Journal of Sleep Research, e12820. Advance online publication. https://doi.org/10.1111/jsr.12820

Giustino, T. F., Fitzgerald, P. J., \& Maren, S. (2016). Fear expression suppresses medial prefrontal cortical firing in rats. PLOS ONE, 11(10), e0165256. https://doi.org/10.1371 /journal.pone.0165256

Golkar, A., Tjaden, C., \& Kindt, M. (2017). Vicarious extinction learning during reconsolidation neutralizes fear memory. Behaviour Research and Therapy, 92, 87-93. https://doi.org/10.1016/j.brat.2017.02.004

Greene, E., \& Greene, A. (1977). Beyond biofeedback. New York, NY: Dell Press.

Harnes, J. M. (2010, August 27). Rapid resolution therapy [Internet Blog]. Retrieved from https://www.army.mil/article /44335/rapid_resolution_therapy

Health, P. B. (2010, June 15). PTSD in veterans linked to dementia in later life [Internet Blog]. Retrieved from https://www.promisesbehavioralhealth.com/ trauma-ptsd/ptsdin-veterans-linked-to-dementia-in-later-life/

Helpman, L., Marin, M.-F., Papini, S., Zhu, X., Sullivan, G. M., Schneier, F., ... Neria, Y. (2016). Neural changes in extinction recall following prolonged exposure treatment for PTSD: A longitudinal fMRI study. Neurolmage: Clinical, 12, 715-723. https://doi.org/10.1016/j.nicl.2016.10.007

Herrington, J. D., Heller, W., Mohanty, A., Engels, A. S., Banich, M. T., Webb, A. G., \& Miller, G. A. (2010). Localization of asymmetric brain function in emotion and depression. Psychophysiology, 47(3), 442-454. https://doi.org/10.1111 /j.1469-8986.2009.00958.x

Isik, B. K., Esen, A., Büyükerkmen, B., Kilinç, A., \& Menziletoglu, D. (2017). Effectiveness of binaural beats in reducing preoperative dental anxiety. The British Journal of Oral and Maxillofacial Surgery, 55(6), 571-574. https://doi.org/10.1016 /j.bjoms.2017.02.014

Kaiser, D. A. (2006). What is quantitative EEG? Journal of Neurotherapy, 10(4), 37-52. https://doi.org/10.1300 /J184v10n04 05

Kaplan, D. M., Smith, T., \& Coons, J. (1995). A validity study of the subjective unit of discomfort (SUD) score. Measurement and Evaluation in Counseling and Development, 27(4), 195199. Retrieved from https://eric.ed.gov/?id=EJ506232

Kibler, J. L. (2009). Posttraumatic stress and cardiac disease risk. Journal of Trauma \& Dissociation, 10(2), 135-150. https://doi.org/10.1080/15299730802624577

Krystal, J. H., Davis, L. L., Neylan, T. C., Raskind, M. A., Schnurr, P. P., Stein, M. B., ... Huang, G. D. (2017). It is time to address the crisis in the pharmacotherapy of posttraumatic stress disorder: A consensus statement of the PTSD Psychopharmacology Working Group. Biological Psychiatry, 82(7), e51-e59. https://doi.org/10.1016 /j.biopsych.2017.03.007

Laird, A. R., Fox, P. M., Eickhoff, S. B., Turner, J. A., Ray, K. L., McKay, D. R., ... Fox, P. T. (2011). Behavioral interpretations of intrinsic connectivity networks. Journal of Cognitive Neuroscience, 23(12), 4022-4037. https://doi.org/10.1162 /jocn_a_00077

Lake, J. (2015). The integrative management of PTSD: A review of conventional and CAM approaches used to prevent and treat PTSD with emphasis on military personnel. Advances in Integrative Medicine, 2(1), 13-23. https://doi.org/10.1016 /j.aimed.2014.10.002

Lande, R. G., Williams, L. B., Francis, J. L., Gragnani, C., \& Morin, M. L. (2010). Efficacy of biofeedback for post-traumatic stress disorder. Complementary Therapeutic Medicine, 18(6), 256-259. https://doi.org/10.1016/j.ctim.2010.08.004

Lanius, R. A., Frewen, P. A., Tursich, M., Jetly, R., \& McKinnon, M. C. (2015). Restoring large-scale brain networks in PTSD and related disorders: A proposal for neuroscientificallyinformed treatment interventions. European Journal of Psychotraumatology, 6(1), 27313-27324. https://doi.org /10.3402/ejpt.v6.27313

Lawlis, F. (2006). About the BAUD [Website]. Retrieved from http://www.baudtherapy.com/about.html

Lawlis, F. (2010). An international clinical study of the sonic disruption of sympathetic arousal principle as designed in the BAUD device. Unpublished manuscript. Retrieved from http://www.baudenergetics.com/images/study\%20for\%20soni c\%20disruptor\%20in\%20int'l\%20study.pdf

Lawlis, F., (2011). The PTSD Breakthrough: The Revolutionary, Science-Based Compass RESET Program. Naperville, IL: Sourcebooks.

LeCron, L. M. (1964). Self-Hypnotism: The technique and its use in daily living. New York, NY: Penguin Books, Ltd.

Levine, P. A., \& Frederick, A. (1997). Waking the tiger: Healing trauma (1st ed.). Berkeley, CA: North Atlantic Books.

Lindenfeld, G., \& Bruursema, L. R. (2015). Resetting the fear switch in PTSD: A novel treatment using acoustical neuromodulation to modify memory reconsolidation. Unpublished manuscript. Retrieved from https://www.academia.edu/12532059/Resetting_the_Fear_S witch_in_PTSD_A_Novel_Treatment_Using_Acoustical_Neur omodulation_to_Modify_Memory_Reconsolidation

Lindenfeld, G., \& Rozelle, G. (2015). PTSD: Brain on fire. A RESET Therapy (QEEG) brain map analysis case study of an Afghanistan combat veteran. Unpublished manuscript. Retrieved from https://www.academia.edu/19524765 /PTSD Brain_On Fire

Lindenfeld, G., Rozelle, G., Soutar, R., Hummer, J., \& Sutherland, M. (2019, Winter). Post-traumatic stress remediated: A study of eight combat veterans. New Mind Journal. Retrieved from http://nmindjournal.com/post-traumatic-stress-remediated-apilot-study-of-8-combat-veterans/

Lipov, E., Tukan, A., \& Candido, K. (2018). It is time to look for new treatments for posttraumatic stress disorder: Can sympathetic system modulation be an answer? Biological Psychiatry, $\quad 84(2), \quad$ e17-e18. https://doi.org/10.1016 /j.biopsych.2017.07.026

Logue, M. W., van Rooij, S. J. H., Dennis, E. L., Davis, S. L., Hayes, J. P., Stevens, J. S., ... Morey, R. A. (2018). Smaller hippocampal volume in posttraumatic stress disorder: A multisite ENIGMA-PGC study: Subcortical volumetry results from posttraumatic stress disorder consortia. Biological Psychiatry, 83(3), 244-253. https://doi.org/10.1016 /j.biopsych.2017.09.006

Luo, Y., Fernández, G., Hermans, E., Vogel, S., Zhang, Y., Li, H., \& Klumpers, F. (2018). How acute stress may enhance subsequent memory for threat stimuli outside the focus of 
attention: DLPFC-amygdala decoupling. Neurolmage, 171, 311-322. https://doi.org/10.1016/j.neuroimage.2018.01.010

Maren, S., \& Holmes, A. (2016). Stress and fear extinction. Neuropsychopharmacology, 41(1), 58-79. https://doi.org /10.1038/npp.2015.180

Martone, G. (2019). Can taming inflammation help reduce aggression? Current Psychiatry, 18(2), 49-50.

McGaugh, J. L. (2000). Memory: A century of consolidation. Science, 287(5451), 248-251. https://doi.org/10.1126 /science.287.5451.248

McGilchrist, I. (2009). The master and his emissary: The divided brain and the making of the western world. New Haven, CT: Yale University Press.

McLeay, S. C., Harvey, W. M., Romaniuk, M. N. M., Crawford, D. H. G., Colquhoun, D. M., Young, R. M., ... Lawford, B. R. (2017). Physical comorbidities of post-traumatic stress disorder in Australian Vietnam War veterans. The Medical Journal of Australia, 206(6), 251-257. https://doi.org/10.5694 /mja16.00935

Mellon, S. H., Gautam, A., Hammamieh, R., Jett, M., \& Wolkowitz, O. M. (2018). Metabolism, metabolomics, and inflammation in posttraumatic stress disorder. Biological Psychiatry, 83(10), 866-875. https://doi.org/10.1016/j.biopsych.2018.02.007

Menon, V., (2015). Salience network. In A. W. Toga (Ed.), Brain mapping: An encyclopedic reference (Vol. 2, pp. 597-611). New York, NY: Academic Press/Elsevier. https://doi.org /10.1016/B978-0-12-397025-1.00052-X

Merabet, L. B., \& Pascual-Leone, A. (2010). Neural reorganization following sensory loss: The opportunity of change. Nature Reviews Neuroscience, 11, 44-52. https://doi.org/10.1038/nrn2758

Miller, J. C., \& Lindenfeld, G. (2017, May). Auditory stimulation therapy for PTSD. Conference presentation presented at the 88th Annual Scientific Meeting of the Aerospace Medical Association, Denver, CO. Retrieved from https://www.academia.edu/32861964/Auditory_Stimulation_T herapy_for_PTSD

Miller, M. W., Lin, A. P., Wolf, E. J., \& Miller, D. R. (2018). Oxidative stress, inflammation, and neuroprogression in chronic PTSD. Harvard Review of Psychiatry, 26(2), 57-69. http://dx.doi.org/10.1097/HRP.0000000000000167

Monfils, M.-H., Cowansage, K. K., Klann, E., \& LeDoux, J. E. (2009). Extinction-reconsolidation boundaries: Key to persistent attenuation of fear memories. Science, 324(5929), 951-955. https://doi.org/10.1126/science. 1167975

Monson, C. M., \& Shnaider, P. (2014). Concise guides on trauma care book series. Treating PTSD with cognitive-behavioral therapies: Interventions that work. Washington, DC: American Psychological Association.

Moore, J. P., Trudeau, D. L., Thuras, P. D., Rubin, Y., Stockley, H., \& Diamond, T. (2000). Comparison of alpha-theta, alpha, and EMG neurofeedback in the production of alpha-theta crossover and the occurrence of visualizations. Journal of Neurotherapy, 4(1), 29-42. https://doi.org/10.1300 /J184v04n01_04

Nagpal, M. L., Gleichauf, K., \& Ginsberg, J. P. (2013). Metaanalysis of heart rate variability as a psychophysiological indicator of posttraumatic stress disorder. Journal of Trauma \& Treatment, 3, 182. https://doi.org/10.4172/21671222.1000182

National Institute of Mental Health (NIMH; 2019). Post-traumatic stress disorder [Website]. Retrieved from https://www.nimh.nih.gov/health/topics/post-traumatic-stressdisorder-ptsd/index.shtml

NeuroGuide Deluxe [Computer software]. (n.d.). Largo, FL: Applied Neuroscience, Inc.

Noohi, S., Miraghaie, A. M., Arabi, A., \& Nooripour, R. (2017). Effectiveness of neuro-feedback treatment with alpha/theta method on PTSD symptoms and their executing function. Biomedical Research, 28(5), 2019-2027. Retrieved from https://pdfs.semanticscholar.org/a07b/8c71fe8556abdca86ed e93b3179f30f8e220.pdf

Orr, S. P., Metzger, L. J., Lasko, N. B., Macklin, M. L., Peri, T., \& Pitman, R. K. (2000). De novo conditioning in traumaexposed individuals with and without posttraumatic stress disorder. Journal of Abnormal Psychology, 109(2), 290-298. https://doi.org/10.1037/0021-843X.109.2.290

Orr, S. P., Meyerhoff, J. L., Edwards, J. V., \& Pitman, R. K. (1998). Heart rate and blood pressure resting levels and responses to generic stressors in Vietnam veterans with posttraumatic stress disorder. Journal of Traumatic Stress, 11(1), 155-164. https://doi.org/10.1023/A:1024421502881

Othmer, S., Othmer S. F., \& Kaiser, D. A. (1999). EEG biofeedback: An emerging model for its global efficacy. In J. R. Evans \& A. Abarbanel (Eds.), Introduction to quantitative EEG and neurofeedback, (1st ed., pp. 243-310). San Diego, CA: Academic Press.

Pascual-Marqui R. D., Lehmann, D., Koukkou, M., Kochi, K., Anderer, P., Saletu, B., ... Kinoshita, T. (2011). Assessing interactions in the brain with exact low-resolution electromagnetic tomography. Philosophical Transactions of the Royal Society A: Mathematical, Physical and Engineering Sciences, 369(1952), 3768-3784. https://doi.org/10.1098 /rsta.2011.0081

Peniston, E. G., \& Kulkosky, P. J. (1989). Alpha-theta brainwave training and beta-endorphin levels in alcoholics. Alcoholism: Clinical \& Experimental Research, 13(2), 271-279. https://doi.org/10.1111/j.1530-0277.1989.tb00325.x

Peniston, E., \& Kulkosky, P. (1991). Alpha-theta brainwave neurofeedback for veterans with combat-related post-traumatic stress disorder. Medical Psychotherapy, 4, 47-60.

Peniston, E. G., Marrinan, D. A., Deming, W. A., \& Kulkosky, P. J. (1993). EEG alpha-theta brainwave synchronization in Vietnam theater veterans with combat-related post-traumatic stress disorder and alcohol abuse. Advances in Medical Psychotherapy, 6, 37-50.

Penix, E. A., Kim, P. Y., Wilk, J. E., \& Adler, A. B. (2019). Secondary traumatic stress in deployed healthcare staff. Psychological Trauma: Theory, Research, Practice, and Policy, 11(1), 1-9. https://doi.org/10.1037/tra0000401

Pfitzer, B. E. (2008). A step towards a broader understanding of complex traumatization in victims of crime (Doctoral dissertation). Retrieved from https://digital.library.adelaide.edu.au/dspace/bitstream/2440/5 6420/8/02main.pdf

Polak, A. R., Witteveen, A. B., Denys, D., \& Olff, M. (2015), Breathing biofeedback as an adjunct to exposure in cognitive behavioral therapy hastens the reduction of PTSD symptoms: A pilot study. Applied Psychophysiology and Biofeedback, 40(1), 25-31. https://doi.org/10.1007/s10484-015-9268-y

Posner, M. I., \& Raichle, M. E. (1994). Images of mind (1st ed., Scientific American Library). New York, NY: W. H. Freeman \& Co.

Psychological Health Center of Excellence (PHCoE; 2018). Biofeedback for posttraumatic stress disorder. [Website] Retrieved from https://www.pdhealth.mil/sites/default/files /images/docs/biofeedback_for_ptsd.pdf

Putnam, J. (2000). The effects of brief, eyes-open alpha brain wave training with audio and video relaxation induction on the EEG of 77 Army reservists. Journal of Neurotherapy, 4(1), 17-28. https://doi.org/10.1300/J184v04n01_03

Pyne, J. M., Constans, J. I., Wiederhold, M. D., Gibson, D. P., Kimbrell, T., Kramer, T. L., ... McCune, T. R. (2016). Heart rate variability: Pre-deployment predictor of post-deployment PTSD symptoms. Biological Psychology, 121(A), 91-98. https://doi.org/10.1016/j.biopsycho.2016.10.008

Rastegar, N., Dolatshahi, B., \& Dogahe, E. R. (2016). The effect of neurofeedback training on increasing sustained attention in veterans with posttraumatic stress disorder. Journal of 
Practice in Clinical Psychology, 4(2), 97-104. https://doi.org/10.15412/J.JPCP.06040204

Rauch, S. L., Shin, L. M., \& Phelps, E. A. (2006). Neurocircuitry models of posttraumatic stress disorder and extinction: Human neuroimaging research-Past, present, and future. Biological Psychiatry, 60(4), 376-382. https://doi.org/10.1016 /j.biopsych.2006.06.004

Rosenthal, M. (2015). The science behind PTSD symptoms: How trauma changes the brain [Internet Blog]. Retrieved from https://psychcentral.com/blog/the-science-behind-ptsdsymptoms-how-trauma-changes-the-brain/

Ryder, A. L., Azcarate, P. M., \& Choen, B. E. (2018). PTSD and physical health. Current Psychiatry Reports, 20(12), 116. http://dx.doi.org/10.1007/s11920-018-0977-9

Schaefer, M. T., \& Olson, D. H. (1981). Assessing intimacy: The PAIR inventory. Journal of Marital and Family Therapy, 7(1), 47-60. https://doi.org/10.1111/j.1752-0606.1981.tb01351.x

Schmidt, L. J., Belopolsky, A. V., \& Theeuwes, J. (2015). Attentional capture by signals of threat. Cognition and Emotion, 29(4), 687-694. https://doi.org/10.1080 /02699931.2014.924484

Schroyens, N., Beckers, T., \& Kindt, M. (2017). In search for boundary conditions of reconsolidation: A failure of fear memory interference. Frontiers in Behavioral Neuroscience, 11, 65. https://doi.org/10.3389/fnbeh.2017.00065

Sewak, R., \& Spielholz, N. I. (2018). Relapse prevention: Using sound to reduce the probability of recidivism and suffering following detoxification. Medical Hypotheses, 118, 84-91. https://doi.org/10.1016/j.mehy.2018.06.023

Smith, W. D. (2008). The effect of neurofeedback training on PTSD symptoms of depression and attention problems among military veterans (Doctoral dissertation). Retrieved from https://search.proquest.com/openview /8e2d34de1edc5f3d730134cff05d6698/1?pqorigsite $=$ gscholar $\& \mathrm{cbl}=18750 \&$ diss $=y$

Soares, J. M., Marques, P., Magalhães, R., Santos, N. C., \& Sousa, N. (2014). Brain structure across the lifespan: The influence of stress and mood. Frontiers in Aging $\begin{array}{llll}\text { Neuroscience, } & 6, & 330 . & \mathrm{https} / / / \mathrm{doi} . \text { org/10.3389 }\end{array}$ /fnagi.2014.00330

Soutar, R. (in press). Holistic neurointegration: The new mind model-A bio-psycho-social qEEG guided neurofeedback method. Roswell, GA: New Mind Academy.

Soutar, R., Hopson, J., \& Longo, R. (2016, Winter). Correlating oxidative stress and qEEG. New Mind Journal. Retrieved from www.nmindjournal.com/oxidative-stress-qeeg/

Speer, K., Upton, D., Semple, S., \& McKune, A. (2018). Systemic low-grade inflammation in post-traumatic stress disorder: A systematic review. Journal of Inflammation Research, 11, 111-121. https://doi.org/10.2147/JIR.S155903

Sporns, O. (2010). Networks of the brain. Cambridge, MA: The MIT Press.

Sumner, J. A., Chen, Q., Roberts, A. L., Winning, A., Rimm, E. B., Gilsanz, P., ... Kubzansky, L. D. (2017). Cross-sectional and longitudinal associations of chronic posttraumatic stress disorder with inflammatory and endothelial function markers in women. Biological Psychiatry, 82(12), 875-884. https://doi.org/10.1016/j.biopsych.2017.06.020

Sung, H.-C., Lee, W.-L., Li, H.-M., Lin, C.-Y., Wu, Y.-Z., Wang, J.J., \& Li, T.-L. (2017). Familiar music listening with binaural beats for older people with depressive symptoms in retirement homes. Neuropsychiatry, 7(4), 347-353. https://doi.org/10.4172/Neuropsychiatry. 1000221

Swingle, P. G. (2008). Biofeedback for the brain: How neurotherapy effectively treats depression, $A D H D$, autism, and more. New Brunswick, NJ: Rutgers University Press.

Tan, G., Dao, T. K., Farmer, L., Sutherland, R. J., \& Gervitz, R. (2011). Heart rate variability (HRV) and posttraumatic stress disorder (PTSD): A pilot study. Applied Psychophysiology and Biofeedback, 36(1), 27-35. https://doi.org/10.1007 /s10484-010-9141-y

Tan, G., Wang, P., \& Ginsberg, J. (2013). Heart rate variability and posttraumatic stress disorder. Biofeedback, 41(3), 131135. https://doi.org/10.5298/1081-5937-41.3.05

Tanielian, T., \& Jaycox, L. H. (Eds., 2008). Invisible wounds of war: Psychological and cognitive injuries, their consequences, and services to assist recovery. Santa Monica, CA: RAND Corporation. Retrieved from https://www.rand.org/pubs /monographs/MG720.html

Thatcher, R. W. (2011). Neuropsychiatry and quantitative EEG in the 21st Century. Neuropsychiatry, 1(5), 495-514. https://doi.org/10.2217/npy. 11.45

Thatcher, R. W., North, D., \& Biver, C. (2005). Evaluation and validity of a LORETA normative EEG database. Clinical EEG and Neuroscience, 36(2), 116. https://doi.org/10.1177 $/ 155005940503600211$

Todder, D., Levine, J., Abujumah, A., Mater, M., Cohen, H., \& Kaplan, Z. (2012). The quantitative electroencephalogram and the low-resolution electrical tomographic analysis in posttraumatic stress disorder. Clinical EEG and Neuroscience, 43(1), 48-53. https://doi.org/10.1177 $/ 1550059411428716$

van der Kolk, B. A., McFarlane, A. C., \& Weisaeth, L. (Eds., 1996). Traumatic stress: The effects of overwhelming experience on mind, body, and society (1st ed.). New York, NY: Guilford Press.

Wahbeh, H., \& Oken, B. S. (2013). Peak high-frequency HRV and peak alpha frequency higher in PTSD. Applied Psychophysiology and Biofeedback, 38(1), 57-69. https://doi.org/10.1007/s10484-012-9208-z

Weathers, F. W., Blake, D. D., Schnurr, P. P., Kaloupek, D. G., Marx, B. P., \& Keane, T. M. (2013). The ClinicianAdministered PTSD Scale for DSM-5 (CAPS-5). [Assessment]. Available from http://www.ptsd.va.gov

White, E. K., Groeneveld, K. M., Tittle, R. K., Bolhuis, N. A., Martin, R. E., Royer, T. G., \& Fotuhi, M. (2017). Combined neurofeedback and heart rate variability training for individuals with symptoms of anxiety and depression: A retrospective study. NeuroRegulation, 4(1), 37-55. https://doi.org/10.15540/nr.4.1.37

Widholm, J. J. (2010). Extinction learning as a model of drug treatment and relapse: A behavioral overview. The Open Addiction Journal, 3(1), 57-62. https://doi.org/10.2174 /1874941001003010057

Received: April 19, 2019

Accepted: June 13, 2019

Published: June 26, 2019 